\title{
Global Solutions to the Coupled Chemotaxis-Fluid Equations
}

\author{
RENJUn DUAN \\ Johann Radon Institute for Computational and Applied Mathematics \\ Austrian Academy of Sciences \\ Altenbergerstrasse 69, A-4040 Linz, Austria \\ Alexander Lorz, Peter Markowich \\ Department of Applied Mathematics and Theoretical Physics \\ University of Cambridge, Wilberforce Road \\ Cambridge CB3 0WA, UK
}

May 25, 2010

\begin{abstract}
In this paper, we are concerned with a model arising from biology, which is a coupled system of the chemotaxis equations and the viscous incompressible fluid equations through transport and external forcing. The global existence of solutions to the Cauchy problem is investigated under certain conditions. Precisely, for the Chemotaxis-Navier-Stokes system over three space dimensions, we obtain global existence and rates of convergence on classical solutions near constant states. When the fluid motion is described by the simpler Stokes equations, we prove global existence of weak solutions in two space dimensions for cell density with finite mass, first-order spatial moment and entropy provided that the external forcing is weak or the substrate concentration is small.
\end{abstract}

Keywords: chemotaxis-fluid interaction; chemotaxis; Stokes equations; global solution; energy method; a priori estimates.

\section{Contents}

1 Introduction $\quad 2$

2 The coupled Chemotaxis-Navier-Stokes equations 5

2.1 Global classical solutions near constant states . . . . . . . . . . . . . . . . . . 5

2.2 Uniform a priori estimates . . . . . . . . . . . . . . . . . . . . . . . . . 7

2.3 Proof of local and global existence . . . . . . . . . . . . . . . . . . . . . . . 10

2.4 Proof of rates of convergence . . . . . . . . . . . . . . . . . . . . . . . 14

3 The coupled Chemotaxis-Stokes equations $\quad 18$

3.1 Global weak solutions with large data . . . . . . . . . . . . . . . . . . 18

3.2 Uniform a priori estimates . . . . . . . . . . . . . . . . . . . . . . . . . 20

3.3 Proof of global existence . . . . . . . . . . . . . . . . . . . . . . . 27 


\section{Introduction}

Chemotaxis is a biological process, in which cells (e.g. bacteria) move towards a chemically more favorable environment. For example, bacteria often swim towards higher concentration of oxygen to survive. The chemical substrate can be produced or consumed by the cells, where we are only interested in the latter in this paper which corresponds to the repulsive case for the substrate. A typical model describing chemotaxis are the Keller-Segel equations derived by Keller and Segel [21] which have become one of the best-studied models in mathematical biology. On the other hand, in nature cells often live in a viscous fluid so that cells and chemical substrates are also transported with fluid, and meanwhile the motion of the fluid is under the influence of gravitational forcing generated by aggregation of cells. Generally, the motion of the fluid is determined by the well-known incompressible Navier-Stokes equations or Stokes equations. Thus, this kind of cell-fluid interaction becomes more complicated since it not only consists of chemotaxis and diffusion, but also includes transport and viscous fluid dynamics. In particular, it is interesting and important in biology to study some phenomenon of sedimentation on the basis of the coupled cell-fluid model.

Recently, to describe the coupled biological phenomena mentioned above, the authors in [30] proposed the following model:

$$
\left\{\begin{array}{l}
\partial_{t} n+u \cdot \nabla n=\delta \Delta n-\nabla \cdot(\chi(c) n \nabla c), \\
\partial_{t} c+u \cdot \nabla c=\mu \Delta c-k(c) n \\
\partial_{t} u+u \cdot \nabla u+\nabla P=\nu \Delta u-n \nabla \phi, \\
\nabla \cdot u=0, \quad t>0, x \in \Omega .
\end{array}\right.
$$

Here, the unknowns are $n=n(t, x): \mathbb{R}^{+} \times \Omega \rightarrow \mathbb{R}^{+}, c=c(t, x): \mathbb{R}^{+} \times \Omega \rightarrow \mathbb{R}^{+}, u(t, x): \mathbb{R}^{+} \times \Omega \rightarrow \mathbb{R}^{3}$ or $\mathbb{R}^{2}$ and $P=P(t, x): \mathbb{R}^{+} \times \Omega \rightarrow \mathbb{R}$, denoting the cell density, substrate concentration, velocity and pressure of the fluid, respectively. $\Omega \subset \mathbb{R}^{3}$ or $\mathbb{R}^{2}$ is a spatial domain where the cells and the fluid move and interact. Constants $\delta, \mu$ and $\nu$ are the corresponding diffusion coefficients for the cells, substrate and fluid. $\chi(c)$ is the chemotactic sensitivity and $k(c)$ is the consumption rate of the substrate by the cells. $\phi=\phi(t, x)$ is a given potential function. We remark that the model (1.1) has been compared numerically with the experiment in [30], and it was also used in [27]. Similar models were investigated in [28] and [17].

As usual, in order for the system (1.1) to be well-posed, it should be supplemented with some initial conditions

$$
\left.(n, c, u)\right|_{t=0}=\left(n_{0}(x), c_{0}(x), u_{0}(x)\right), \quad x \in \Omega,
$$

and some proper boundary conditions. See [30] and references therein for the discussions on the boundary conditions if $\Omega$ is a bounded domain. In the sequel we shall take $\Omega=\mathbb{R}^{2}$ or $\mathbb{R}^{3}$ in order to consider the well-posedness for the Cauchy problem (1.1)-(1.2) over the whole space. Moreover, we shall also consider the simplified Chemotaxis-Stokes system taking the following form

$$
\left\{\begin{array}{l}
\partial_{t} n+u \cdot \nabla n=\delta \Delta n-\nabla \cdot(\chi(c) n \nabla c), \\
\partial_{t} c+u \cdot \nabla c=\mu \Delta c-k(c) n, \\
\partial_{t} u+\nabla P=\nu \Delta u-n \nabla \phi \\
\nabla \cdot u=0, \quad t>0, x \in \Omega,
\end{array}\right.
$$

where compared with (1.1), the nonlinear convective term $u \cdot \nabla u$ is ignored in the fluid equation of (1.3). 
It can be seen from (1.1) or (1.3) that the coupling of chemotaxis and fluid is realized through both the transport of cells and chemical substrates $u \cdot \nabla n, u \cdot \nabla c$ and the external force $-n \nabla \phi$ exerted on the fluid by cells. Here, for the external force, it can be produced by different physical mechanism such as gravity, centrifugal, electric or magnetic forces. One example in the case of gravity is

$$
\phi=a x_{1},
$$

for a constant $a \in \mathbb{R}$ depending on the ratio of the fluid mass density to the cell density and the gravity acceleration. Another example is the centrifugal force $\phi$ satisfying

$$
0 \leq \phi(x)=\phi(|x|) \rightarrow 0 \text { as }|x| \rightarrow \infty
$$

In this paper, we are only interested in the case of the centrifugal force with $\phi$ decaying in $x$ with some rates at infinity.

Throughout this paper, we also assume the following conditions:

$$
\text { (A) }\left\{\begin{array}{l}
(i) \delta>0, \mu>0, \nu>0 ; \\
(i i) n_{0}(x) \geq 0, c_{0}(x) \geq 0, \nabla \cdot u_{0}(x)=0 \text { for all } x \in \Omega ; \\
\left(\text { iii } \chi(\cdot), k(\cdot) \text { and } \phi(\cdot, \cdot) \text { are smooth with } k(0)=0 \text { and } k^{\prime}(c) \geq 0 \text { for all } c \in \mathbb{R} .\right.
\end{array}\right.
$$

Notice that the above assumption implies that $c$ preserves the nonnegativity of the initial data by the maximum principle, and moreover, $k(c) \geq 0$ holds for $c \geq 0$, that is the case of consumption of chemical substrates. Thus, the interaction force caused by the oxygen is repulsive but not attractive as in the classical Keller-Segel system. Later, we shall point out some special properties that the Keller-Segel system enjoys when $k(c)$ takes the negative sign. We further remark that if the fluid coupling is neglected, i.e. $u=0$, then the system obtained is similar to a drift-diffusion model in the context of semiconductor, see [24].

The aim of this paper is to obtain some global existence results for the Cauchy problem on the above systems (1.1) and (1.3) under the assumption (A) and some additional conditions on the initial data and on the functions $\chi(c), k(c), \phi$. It should be pointed out that these results could also hold over the smooth bounded domain $\Omega$ with homogeneous boundary conditions. Precisely, the first result of this paper is Theorem 2.1 which states the global existence of classical solutions to the Cauchy problem on the system (1.1) and (1.2) over $\Omega=\mathbb{R}^{3}$ provided that the initial datum $\left(n_{0}, c_{0}, u_{0}\right)$ is a small smooth perturbation of the constant state $\left(n_{\infty}, 0,0\right)$ with $n_{\infty} \geq 0$, and the potential function $\phi$ depending on $t, x$ decays in $x$ at infinity uniformly in time. The proof is based on some uniform a priori estimates combined with the local existence as well as the standard continuity argument. Moreover, the timedecay rates in $L^{p}$-norms for perturbations are obtained in Theorem 2.2, where $L^{p}$ energy methods and spectral analysis are used in the proof. Here, the method of proving the optimal time-decay rate by combining the energy estimates and spectral analysis is actually the one recently developed by [11] in the study of the nonlinear Boltzmann equation.

The second result is Theorem 3.1 about the global existence of weak solutions to the Cauchy problem on the system (1.3) with large initial data (1.2) in some functional spaces over $\Omega=\mathbb{R}^{2}$. In fact, the existence of global weak solution is obtained when initial density $n_{0}$ has finite mass, firstorder spatial moment and entropy under the additional condition that the strength of the nonnegative time-independent potential function $\phi$ is weak or $c_{0}$ is small in some sense. The main part in the proof is to derive some uniform a priori estimates by constructing some proper free energy functionals. To explain this more, let us here skip the coupling of fluid, that is to consider the repulsive chemotaxis system in the case of the consumption of chemical substrate:

$$
\left\{\begin{array}{l}
\partial_{t} n=\delta \Delta n-\nabla \cdot(\chi(c) n \nabla c), \\
\partial_{t} c=\mu \Delta c-k(c) n, \quad t>0, x \in \Omega .
\end{array}\right.
$$


Define the instant free energy functional $\mathcal{E}(n(t), c(t))$ by

$$
\mathcal{E}(n(t), c(t))=\int_{\Omega} n \ln n d x+\frac{1}{2} \int_{\Omega}|\nabla \Psi(c)|^{2} d x,
$$

where $\Psi(c)$ is given by

$$
\Psi(c)=\int_{0}^{c}\left(\frac{\chi(s)}{k(s)}\right)^{1 / 2} d s .
$$

Then, for any smooth solution $(n, c)$ to the system (1.4),

$$
\frac{d}{d t} \mathcal{E}(n(t), c(t))+\mathcal{D}(n(t), c(t))=0
$$

holds for any $t \geq 0$, where the dissipation rate $\mathcal{D}(n(t), c(t))$ is given by

$$
\begin{aligned}
\mathcal{D}(n(t), c(t))= & \delta \int_{\Omega} \frac{|\nabla n|^{2}}{n} d x+\int_{\Omega} \frac{\chi^{\prime}(c) k(c)+\chi(c) k^{\prime}(c)}{2 \chi(c)} n|\nabla \Psi|^{2} d x \\
& +\mu \int_{\Omega}\left|\nabla^{2} \Psi-\frac{d}{d c} \sqrt{\frac{k(c)}{\chi(c)}} \nabla \Psi \otimes \nabla \Psi\right|^{2} d x \\
& -\frac{\mu}{2} \int_{\Omega} \frac{d^{2}}{d c^{2}}\left(\frac{k(c)}{\chi(c)}\right)|\nabla \Psi|^{4} d x .
\end{aligned}
$$

Thus, under the condition that

$$
\chi(c)>0, \quad \frac{d}{d c}(\chi(c) k(c))>0, \quad \frac{d^{2}}{d c^{2}}\left(\frac{k(c)}{\chi(c)}\right)<0,
$$

$\mathcal{D}(n(t), c(t))$ becomes a nonnegative functional and hence the free energy $\mathcal{E}(n(t), c(t))$ is non-increasing in time $t$. When the equations are coupled with the fluid, the additional terms from the transport $u \cdot \nabla n, u \cdot \nabla c$ and the force $-n \nabla \phi$ turn out to be controlled by the total dissipation rate $\mathcal{D}_{\text {total }}(n(t), c(t), u(t))$ if the potential function $\phi$ is small in an appropriate sense. Furthermore, the boundedness of the entropy $\|n \ln n\|_{L^{1}}$ in finite time is proved together with the boundedness of firstorder spatial moment $\left\|\sqrt{1+x^{2}} n\right\|_{L^{1}}$. Instead of smallness of external forcing $\nabla \phi$, if it is assumed that $k^{\prime}(0)>0$ and $\left\|c_{0}\right\|_{L^{\infty}}$ is small enough, then by taking proper linear combination, one can also find a non-increasing free energy functional and thus uniform a priori estimates also follow.

Now we mention some work related to issues discussed in this paper. Firstly, since the model system (1.1) is proposed by [30] for the study of the biology of chemotaxis, let us recall some important mathematical characters on the classical chemotaxis system, the Keller-Segel model, for which one of the simple versions takes the form

$$
\left\{\begin{array}{l}
\partial_{t} n=\Delta n-\chi \nabla \cdot(n \nabla c), \\
-\Delta c=n, \quad t>0, x \in \Omega=\mathbb{R}^{2},
\end{array}\right.
$$

where $\chi$ has been supposed to be a positive constant, $k(c) \equiv-1$ takes the minus sign, and other constants are normalized to have unit values. Readers can refer to the surveys [18, 19] for a summary results on the above system and even more general Keller-Segel system. Here, it should be emphasized that compared with the system (1.4) under the assumption (A) which particularly implies $k(c) \geq 0$, the classical Keller-Segel system (1.6) exposes some different phenomena especially such as critical mass and blow-up. Precisely, the elementary computations for (1.6) show

$$
\frac{d}{d t} \int_{\mathbb{R}^{2}}|x|^{2} n(t, x) d x=4\left\|n_{0}\right\|_{L^{1}}\left(1-\frac{\chi\left\|n_{0}\right\|_{L^{1}}}{8 \pi}\right) .
$$


Thus, the situations leading to the different properties of solutions are basically divided into three cases:

$$
\left\|n_{0}\right\|_{L^{1}}<\frac{8 \pi}{\chi} \text { (subcritical), }\left\|n_{0}\right\|_{L^{1}}=\frac{8 \pi}{\chi} \text { (critical), }\left\|n_{0}\right\|_{L^{1}}>\frac{8 \pi}{\chi} \text { (supercritical), }
$$

where under some conditions, solutions to (1.6) blow up in finite time for the supercritical case while solutions exist globally in time for the subcritical case. The further literature on the Keller-Segel model is indeed huge and thus out of the scope of this paper; see recent work $[1,2,5]$ and references therein.

Next, let us mention some work $[31,9,10,29,12,15]$, which are more connected to the results obtained in this paper. The identity (1.5), which plays a key role in the derivation of the uniform $a$ priori estimates, is inspired by the proof of [31] in the case of bounded domain over $\mathbb{R}^{2}$. Notice that from the late proof, the derivation of the identity (1.5) does not depend on the spatial dimension. For a chemotaxis model motivated by angiogenesis, [9] and [10] proved global existence of weak solutions in high space dimensions for the case when $k(c)=c^{m}$ with $m \geq 1$ by controlling the $L^{p}$-norm of density $n(t, x)$. For the same model in one-dimensional space, the global existence of classical solutions was studied in [29] and [12] over the bounded interval of $\mathbb{R}$ when $\chi(c)=c^{-\alpha}$ and $k(c)=c^{m}$ with $\alpha$ and $m$ taking different values, and was later generalized in [15] to the full line $\mathbb{R}$ with $\chi(c)=\frac{1}{1+c}$ and $k(c)=c$.

On the other hand, more attention has recently been focused on some coupled kinetic-fluid system firstly introduced in [4] which describe the interaction of two phases: the disperse phase of particles and dense phase of fluid. For that, refer to $[16,6,26,25,13,3,14]$ for the study of the Vlasov-FokkerPlanck equation coupled with the compressible or incompressible Navier-Stokes or Stokes equations, where the main tool used to prove the global existence of weak solutions or hydrodynamical limit is the existing entropy inequality. It should also be pointed out that the models for chemotaxis can be justified as an asymptotic limit of some kinetic models, cf. $[8,7]$. Thus, it is interesting to establish the asymptotic validity of the coupled chemotaxis-fluid system from the kinetic level [13].

Finally, the fluid dynamic equations such as the Navier-Stokes or Stokes equations have a much longer history, see $[22,23]$ and references therein for the detailed mathematical theory.

The rest of this paper is organized as follows. In Section 2, we consider the chemotaxis system coupled with the Navier-Stokes equations, and we prove global existence and convergence rates of classical solutions near constant states. In Section 3, we prove global existence of weak solutions to the coupled chemotaxis-Stokes equations under two different assumptions for weak forces or small initial concentration of $c$.

\section{The coupled Chemotaxis-Navier-Stokes equations}

\subsection{Global classical solutions near constant states}

As mentioned before, we consider in this section the Cauchy problem on the coupled ChemotaxisNavier-Stokes equations in $\mathbb{R}^{3}$ :

$$
\left\{\begin{array}{l}
\partial_{t} n+u \cdot \nabla n=\delta \Delta n-\nabla \cdot(\chi(c) n \nabla c), \\
\partial_{t} c+u \cdot \nabla c=\mu \Delta c-k(c) n, \\
\partial_{t} u+u \cdot \nabla u+\nabla P=\nu \Delta u-n \nabla \phi, \\
\nabla \cdot u=0, \quad t>0, x \in \mathbb{R}^{3},
\end{array}\right.
$$

with initial data

$$
\left.(n, c, u)\right|_{t=0}=\left(n_{0}(x), c_{0}(x), u_{0}(x)\right), \quad x \in \mathbb{R}^{3},
$$

where it is supposed to hold that

$$
\left(n_{0}(x), c_{0}(x), u_{0}(x)\right) \rightarrow\left(n_{\infty}, 0,0\right) \text { as }|x| \rightarrow \infty,
$$


for some constant $n_{\infty} \geq 0$. The goal of this section is to prove the global existence of classical solutions to the above Cauchy problem when initial data is a small smooth perturbation near the constant steady state $\left(n_{\infty}, 0,0\right)$, and moreover, obtain the rates of convergence of solutions towards this steady state in some functional spaces.

For simplicity of presentation later, let us take change of variables $n=\sigma+n_{\infty}$ and $\bar{P}=P+n_{\infty} \phi$ so that the Cauchy problem (2.1) and (2.2) is reformulated as

$$
\left\{\begin{array}{l}
\partial_{t} \sigma+u \cdot \nabla \sigma-\delta \Delta \sigma=-\nabla \cdot(\chi(c) \sigma \nabla c)-n_{\infty} \nabla \cdot(\chi(c) \nabla c), \\
\partial_{t} c+u \cdot \nabla c-\mu \Delta c+k^{\prime}(0)\left(\sigma+n_{\infty}\right) c=-\left(k(c)-k^{\prime}(0) c\right)\left(\sigma+n_{\infty}\right), \\
\partial_{t} u+u \cdot \nabla u+\nabla \bar{P}-\nu \Delta u=-\sigma \nabla \phi \\
\nabla \cdot u=0, \quad t>0, x \in \mathbb{R}^{3}
\end{array}\right.
$$

with

$$
\left.(\sigma, c, u)\right|_{t=0}=\left(\sigma_{0}(x), c_{0}(x), u_{0}(x)\right) \rightarrow(0,0,0) \text { as }|x| \rightarrow \infty,
$$

where $\sigma_{0}=n_{0}-n_{\infty}$. The first result of this section about the global existence of classical solutions is stated as follows. Here and in the sequel, for simplicity, $\partial^{\alpha}$ means the spatial derivatives $\partial_{x}^{\alpha}$ with multi-index $\alpha, \nabla^{j}$ means all the spatial derivatives of $j$-order for integer $j \geq 0$, and $\|\cdot\|$ always denotes the $L^{2}$-norm without confusion.

Theorem 2.1. Let $n_{\infty} \geq 0$ be a constant, and the assumption $(\mathbf{A})$ hold with $n_{0}(x) \equiv \sigma_{0}(x)+n_{\infty} \geq 0$ for $x \in \mathbb{R}^{3}$, and

$$
\sup _{t, x}(1+|x|)|\phi(t, x)|+\sum_{1 \leq|\alpha| \leq 3} \sup _{t, x}\left|\partial^{\alpha} \phi(t, x)\right|<\infty .
$$

Furthermore, suppose that $\left\|\left(\sigma_{0}, c_{0}, u_{0}\right)\right\|_{H^{3}}$ is sufficiently small. Then, the Cauchy problem (2.3) and (2.4) admits a unique classical solution $(\sigma, c, u)$ satisfying that

$$
n(t, x) \equiv \sigma(t, x)+n_{\infty} \geq 0, c(t, x) \geq 0, t \geq 0, x \in \mathbb{R}^{3},
$$

and there are constants $\lambda>0, C$ such that

$$
\begin{gathered}
\|(\sigma, c, u)(t)\|_{H^{3}}^{2}+\lambda \int_{0}^{t} \int_{\mathbb{R}^{3}}\left(\sigma+n_{\infty}\right)\left[k(c) c+k^{\prime}(0) \sum_{1 \leq|\alpha| \leq 3}\left|\partial^{\alpha} c(s)\right|^{2}\right] d x d s \\
+\lambda \int_{0}^{t}\|\nabla(\sigma, c, u)(s)\|_{H^{3}}^{2} d s \leq C\left\|\left(n_{0}, c_{0}, u_{0}\right)\right\|_{H^{3}}^{2}
\end{gathered}
$$

holds for any $t \geq 0$.

Remark 2.1. The assumption (2.5) in Theorem 2.1 shows that the potential function $\phi(t, x)$ of the external force field depending on both $t$ and $x$ need not be small and also need not decay in time, which is essentially caused by the fact that $\phi(t, x)$ only appears in the linear coupling term $-n \nabla \phi$ in the fluid equation. From the later proof, the spatial decay of $\phi$ with rate $(1+|x|)^{-1}$ can be replaced the integrability condition $\phi \in L^{3}\left(\mathbb{R}^{3}\right)$. Finally, we should emphasize again that the condition in $(\mathbf{A})$ that $k(c)$ is non-decreasing in c plays a key role in obtaining the Lyapunov-type inequality (2.6) for the stability of solution, and as shown in the next section later, it also plays an important role in considering the global existence of weak solutions (cf. Theorem 3.1) or global classical solutions with large smooth data (cf. Proposition 3.1).

The second result of this section is concerned with the time-decay rates of the obtained classical solutions near constant steady states. 
Theorem 2.2. Let $n_{\infty}=0$, and let all conditions in Theorem 2.1 hold. The solution $(\sigma, c, u)$ to the Cauchy problem (2.3)-(2.4) obtained in Theorem 2.1 enjoys the following time-decay estimates. Assume that $\sigma_{0}, c_{0} \in L^{1}\left(\mathbb{R}^{3}\right)$. Then, for any $1 \leq p<\infty$, it holds that

$$
\begin{aligned}
\|\sigma(t)\|_{L^{p}} & \leq C\left\|\sigma_{0}\right\|_{L^{1} \cap L^{p}}(1+t)^{-\frac{3}{2}\left(1-\frac{1}{p}\right)}, \\
\|c(t)\| \|_{L^{p}} & \leq C\left\|c_{0}\right\|_{L^{1} \cap L^{p}}(1+t)^{-\frac{3}{2}\left(1-\frac{1}{p}\right)},
\end{aligned}
$$

for any $t \geq 0$. If it is further assumed that $u_{0} \in L^{q}\left(\mathbb{R}^{3}\right)$ and

$$
\phi \in L^{\infty}\left(\mathbb{R}^{+} ; L^{2 q /(2-q)}\left(\mathbb{R}^{3}\right)\right)
$$

with $1<q<6 / 5$, then it holds that

$$
\|u(t)\| \leq C\left(\left\|u_{0}\right\|_{L^{q} \cap H^{3}}+K_{0}\right)(1+t)^{-\frac{3}{2}\left(\frac{1}{q}-\frac{1}{2}\right)},
$$

for any $t \geq 0$, where $K_{0}$ is defined by

$$
K_{0}=\left\|\left(\sigma_{0}, c_{0}\right)\right\|_{L^{1} \cap H^{3}}+\left\|\sigma_{0}\right\|_{L^{1} \cap L^{2}}\left\|c_{0}\right\|_{L^{1} \cap L^{2}} .
$$

Remark 2.2. Due to time-decay estimates on the high-order derivatives of $(n, c, u)(c f .(2.41)$ and (2.47)) in the proof of Theorem 2.2, the time-decay rates of $L^{\infty}$-norms for $(n, c, u)$ can also follow from Sobolev inequalities. When $n_{\infty}>0$, the proof of Theorem 2.2 can be modified to obtain similar time-decay estimates, which we shall not pursuit in this paper for brevity. For that, a further remark will be given at the end of this section in order to explain it only at the level of linearization.

\subsection{Uniform a priori estimates}

Theorem 2.1 will be proved by combining the local existence and some uniform a priori estimates as well as the continuation argument. In this subsection, we devote ourselves to the proof of uniform $a$ priori estimates. For this purpose, for any $0<T \leq \infty$, let us denote the space

$$
\begin{gathered}
X(0, T)=\left\{(\sigma, c, u): \sigma, c, u \in C^{0}\left([0, T) ; H^{3}\left(\mathbb{R}^{3}\right)\right) \cap C^{1}\left([0, T) ; H^{1}\left(\mathbb{R}^{3}\right)\right)\right. \\
\left.\nabla \sigma, \nabla c, \nabla u \in L^{2}\left([0, T] ; H^{3}\left(\mathbb{R}^{3}\right)\right)\right\} .
\end{gathered}
$$

Lemma 2.1 (a priori estimates). Let all conditions in Theorem 2.1 hold. Suppose that the Cauchy problem (2.3) and (2.4) has a solution $(\sigma, c, u)$ in $X(0, T)$ with

$$
\sup _{0 \leq t \leq T}\|(\sigma, c, u)(t)\|_{H^{3}} \leq \epsilon
$$

for $0<\epsilon \leq 1$. Then $n(t, x) \equiv \sigma(t, x)+n_{\infty} \geq 0, c(t, x) \geq 0$ hold for any $0 \leq t \leq T, x \in \mathbb{R}^{3}$. Furthermore, there are $\epsilon_{0}>0, C_{0}>0$ and $\lambda_{0}>0$ such that for any $\epsilon \leq \epsilon_{0}$,

$$
\begin{gathered}
\|(\sigma, c, u)(t)\|_{H^{3}}^{2}+\lambda_{0} \int_{0}^{t} \int_{\mathbb{R}^{3}}\left(\sigma+n_{\infty}\right)\left[k(c) c+k^{\prime}(0) \sum_{1 \leq|\alpha| \leq 3}\left|\partial^{\alpha} c(s)\right|^{2}\right] d x d s \\
+\lambda_{0} \int_{0}^{t}\|\nabla(\sigma, c, u)(s)\|_{H^{3}}^{2} d s \leq C_{0}\left\|\left(n_{0}, c_{0}, u_{0}\right)\right\|_{H^{3}}^{2}
\end{gathered}
$$

holds for any $0 \leq t \leq T$.

Proof. By the Sobolev imbedding theorem, from (2.10), one has

$$
\sup _{0 \leq t \leq T}\|(\sigma, c, u)\|_{W^{1, \infty}} \leq C \epsilon .
$$


The original equations $(2.1)_{1}-(2.1)_{2}$ can be rewritten as

$$
\begin{aligned}
& \partial_{t} n+\nabla \cdot(\delta \nabla n+n(u+\chi(c) \nabla c))=0 \\
& \partial_{t} c+u \cdot \nabla c=\mu \Delta c-k^{\prime}(\xi) n c
\end{aligned}
$$

where $\xi=\xi(t, x)$ is between 0 and $c(t, x)$. Thus, by the assumption A1, the maximum principle implies

$$
n(t, x) \geq 0, \quad 0 \leq c(t, x) \leq\|c\|_{L^{\infty}} \leq C \epsilon \leq C,
$$

for any $0 \leq t \leq T, x \in \mathbb{R}^{3}$.

To prove (2.11), we divide it into two steps. In the first step, we deal with the zero-order estimates. For $(2.3)_{2}$ on $c$, it holds that

$$
\frac{1}{2} \frac{d}{d t} \int_{\mathbb{R}^{3}} c^{2} d x+\mu \int_{\mathbb{R}^{3}}|\nabla c|^{2} d x+\int_{\mathbb{R}^{3}} k(c) c\left(\sigma+n_{\infty}\right) d x=0 .
$$

For $(2.3)_{1}$ on $n$, it follows from integration by parts and the Cauchy-Schwarz inequality that

$$
\begin{aligned}
\frac{1}{2} \frac{d}{d t} \int_{\mathbb{R}^{3}} \sigma^{2} d x+\frac{\delta}{2} \int_{\mathbb{R}^{3}}|\nabla \sigma|^{2} d x & \leq \frac{1}{\delta}\left(\|n\|_{L_{x}^{\infty}}^{2}+n_{\infty}^{2}\right)\|\chi(c)\|_{L_{x}^{\infty}}^{2} \int_{\mathbb{R}^{3}}|\nabla c|^{2} d x \\
& \leq \frac{C}{\delta}\left(1+n_{\infty}^{2}\right) \max _{0 \leq c \leq C}|\chi(c)|^{2} \int_{\mathbb{R}^{3}}|\nabla c|^{2} d x .
\end{aligned}
$$

For $(2.3)_{3}$ on $u$, one can use the Hardy's inequality [22]

$$
\int_{\mathbb{R}^{3}} \frac{|n|^{2}}{|x|^{2}} d x \leq C\|\nabla n\|^{2}
$$

to get

$$
\frac{1}{2} \frac{d}{d t} \int_{\mathbb{R}^{3}}|u|^{2} d x+\frac{\nu}{2} \int_{\mathbb{R}^{3}}|\nabla u|^{2} d x \leq \frac{C}{\nu}\left(\sup _{t, x}|x||\phi(t, x)|\right)^{2} \int_{\mathbb{R}^{3}}|\nabla n|^{2} d x .
$$

By choosing the constants $d_{1}>0, d_{2}>0$ large enough such that

$$
\frac{d_{1} \mu}{2} \geq \frac{C}{\delta}\left(1+n_{\infty}^{2}\right) \max _{0 \leq c \leq C}|\chi(c)|^{2}, \quad \frac{d_{2} \delta}{4} \geq \frac{C}{\nu}\left(\sup _{t, x}|x||\phi(t, x)|\right)^{2}
$$

the linear combination of (2.13), (2.14) and (2.15) yields

$$
\begin{gathered}
\frac{1}{2} \frac{d}{d t} \int_{\mathbb{R}^{3}}\left(|u|^{2}+d_{2} \sigma^{2}+d_{1} d_{2} c^{2}\right) d x+\frac{\nu}{2} \int_{\mathbb{R}^{3}}|\nabla u|^{2} d x+\frac{d_{2} \delta}{4} \int_{\mathbb{R}^{3}}|\nabla \sigma|^{2} d x \\
+\frac{d_{1} d_{2} \mu}{2} \int_{\mathbb{R}^{3}}|\nabla c|^{2} d x+d_{1} d_{2} \int_{\mathbb{R}^{3}} k(c) c\left(\sigma+n_{\infty}\right) d x \leq 0
\end{gathered}
$$

For the second step, we make estimates on the high-order derivatives of $(\sigma, c, u)$. Take $\alpha$ with $1 \leq|\alpha| \leq 3$. Applying $\partial^{\alpha}$ to $(2.3)_{2}$, multiplying by $\partial^{\alpha} c$ and then integrating, one has

$$
\begin{aligned}
& \frac{1}{2} \frac{d}{d t} \int_{\mathbb{R}^{3}}\left|\partial^{\alpha} c\right|^{2} d x+\mu \int_{\mathbb{R}^{3}}\left|\nabla \partial^{\alpha} c\right|^{2} d x+k^{\prime}(0) \int_{\mathbb{R}^{3}}\left(\sigma+n_{\infty}\right)\left|\partial^{\alpha} c\right|^{2} d x \\
& =\sum_{\beta<\alpha} C_{\beta}^{\alpha} \int_{\mathbb{R}^{3}} \partial^{\alpha-\beta} u \cdot \nabla \partial^{\alpha} c \partial^{\beta} c d x-k^{\prime}(0) \sum_{\beta<\alpha} C_{\beta}^{\alpha} \int_{\mathbb{R}^{3}} \partial^{\alpha-\beta} \sigma \partial^{\beta} c \partial^{\alpha} c d x \\
& \quad-\int_{\mathbb{R}^{3}} \partial^{\alpha}\left[\left(k(c)-k^{\prime}(0) c\right) \sigma\right] \partial^{\alpha} c d x-n_{\infty} \int_{\mathbb{R}^{3}} \partial^{\alpha}\left(k(c)-k^{\prime}(0) c\right) \partial^{\alpha} c d x,
\end{aligned}
$$


where from the Cauchy-Schwarz inequality, (2.10) and (2.12), the right hand side is bounded by

$$
C \epsilon \sum_{1 \leq|\beta| \leq 4} \int_{\mathbb{R}^{3}}\left|\partial^{\alpha} c\right|^{2} d x .
$$

Then, since $\epsilon>0$ can be small enough, after summation over $1 \leq|\alpha| \leq 3$, one has

$$
\begin{aligned}
\frac{1}{2} \frac{d}{d t} \sum_{1 \leq|\alpha| \leq 3} \int_{\mathbb{R}^{3}}\left|\partial^{\alpha} c\right|^{2} d x & +\frac{\mu}{2} \sum_{1 \leq|\alpha| \leq 3} \int_{\mathbb{R}^{3}}\left|\nabla \partial^{\alpha} c\right|^{2} d x \\
& +k^{\prime}(0) \sum_{1 \leq|\alpha| \leq 3} \int_{\mathbb{R}^{3}}\left(\sigma+n_{\infty}\right)\left|\partial^{\alpha} c\right|^{2} d x \leq C \epsilon\|\nabla c\|^{2} .
\end{aligned}
$$

Similarly, it follows from $(2.3)_{1}$ that

$$
\begin{aligned}
& \frac{1}{2} \frac{d}{d t} \int_{\mathbb{R}^{3}}\left|\partial^{\alpha} \sigma\right|^{2} d x+\delta \int_{\mathbb{R}^{3}}\left|\nabla \partial^{\alpha} \sigma\right|^{2} d x \\
& =\sum_{\beta<\alpha} C_{\beta}^{\alpha} \int_{\mathbb{R}^{3}} \partial^{\alpha-\beta} u \cdot \nabla \partial^{\alpha} \sigma \partial^{\beta} \sigma d x+\int_{\mathbb{R}^{3}} \partial^{\alpha}(\chi(c) \sigma \nabla c) \cdot \nabla \partial^{\alpha} \sigma d x \\
& \quad+n_{\infty} \int_{\mathbb{R}^{3}} \partial^{\alpha}(\chi(c) \nabla c) \cdot \nabla \partial^{\alpha} \sigma d x
\end{aligned}
$$

which is bounded by

$$
\frac{\delta}{4} \int_{\mathbb{R}^{3}}\left|\nabla \partial^{\alpha} \sigma\right|^{2} d x+C \epsilon^{2} \sum_{1 \leq|\beta| \leq 4} \int_{\mathbb{R}^{3}}\left|\partial^{\beta} \sigma\right|^{2} d x+C\left(n_{\infty}^{2}+1\right) \sum_{|\beta| \leq 3} \int_{\mathbb{R}^{3}}\left|\nabla \partial^{\beta} c\right|^{2} d x .
$$

Then one has

$$
\begin{aligned}
& \frac{1}{2} \frac{d}{d t} \sum_{1 \leq|\alpha| \leq 3} \int_{\mathbb{R}^{3}}\left|\partial^{\alpha} \sigma\right|^{2} d x+\frac{\delta}{2} \sum_{1 \leq|\alpha| \leq 3} \int_{\mathbb{R}^{3}}\left|\nabla \partial^{\alpha} \sigma\right|^{2} d x \\
& \leq C \epsilon^{2} \int_{\mathbb{R}^{3}}|\nabla \sigma|^{2} d x+C\left(n_{\infty}^{2}+1\right) \sum_{|\beta| \leq 3} \int_{\mathbb{R}^{3}}\left|\nabla \partial^{\beta} c\right|^{2} d x .
\end{aligned}
$$

For $\partial^{\alpha} u$, it follows from $(2.3)_{3}$ that

$$
\begin{aligned}
& \frac{1}{2} \frac{d}{d t} \int_{\mathbb{R}^{3}}\left|\partial^{\alpha} u\right|^{2} d x+\nu \int_{\mathbb{R}^{3}}\left|\nabla \partial^{\alpha} u\right|^{2} d x \\
& =\sum_{\beta<\alpha} \sum_{i j} C_{\beta}^{\alpha} \int_{\mathbb{R}^{3}} \partial^{\alpha-\beta} u_{i} \partial^{\alpha} \partial_{i} u_{j} \partial^{\beta} u_{j} d x+\int_{\mathbb{R}^{3}} \partial^{\alpha}(\nabla \sigma \phi) \cdot \partial^{\alpha} u d x,
\end{aligned}
$$

which is bounded by

$$
\begin{aligned}
\frac{\nu}{4} \int_{\mathbb{R}^{3}}\left(\left|\nabla \partial^{\alpha} u\right|^{2}+\left|\partial^{\alpha} u\right|^{2}\right) d x & +C \epsilon^{2} \sum_{1 \leq|\beta| \leq 3} \int_{\mathbb{R}^{3}}\left|\partial^{\beta} u\right|^{2} d x \\
& +C \sup _{t}\|\phi(t)\|_{W_{x}^{3}, \infty}^{2} \sum_{|\beta| \leq 3} \int_{\mathbb{R}^{3}}\left|\nabla \partial^{\beta} \sigma\right|^{2} d x
\end{aligned}
$$

Thus one has

$$
\begin{aligned}
& \frac{1}{2} \frac{d}{d t} \sum_{1 \leq|\alpha| \leq 3} \int_{\mathbb{R}^{3}}\left|\partial^{\alpha} u\right|^{2} d x+\frac{\nu}{2} \sum_{1 \leq|\alpha| \leq 3} \int_{\mathbb{R}^{3}}\left|\nabla \partial^{\alpha} u\right|^{2} d x \\
& \leq\left(\frac{\nu}{4}+C \epsilon^{2}\right) \int_{\mathbb{R}^{3}}|\nabla u|^{2} d x+C \sup _{t}\|\phi(t)\|_{W_{x}^{3}, \infty}^{2} \sum_{|\beta| \leq 3} \int_{\mathbb{R}^{3}}\left|\nabla \partial^{\beta} \sigma\right|^{2} d x
\end{aligned}
$$


Now, by choosing $d_{3}, d_{4}$ large enough such that

$$
\frac{d_{3}}{4} \geq C \sup _{t}\|\phi(t)\|_{W_{x}^{3, \infty}}^{2}, \quad \frac{\mu d_{4}}{4} \geq C d_{3}\left(n_{\infty}^{2}+1\right),
$$

then the linear combination $(2.17) \times d_{4}+\left[(2.18) \times d_{3}+(2.19)\right]$ leads to

$$
\begin{aligned}
\frac{1}{2} \frac{d}{d t} \sum_{1 \leq|\alpha| \leq 3} C_{\alpha} \int_{\mathbb{R}^{3}}\left|\partial^{\alpha}(\sigma, c, u)\right|^{2} d x+\lambda \sum_{2 \leq|\alpha| \leq 4} \int_{\mathbb{R}^{3}}\left|\partial^{\alpha}(\sigma, c, u)\right|^{2} d x \\
+\lambda k^{\prime}(0) \sum_{1 \leq|\alpha| \leq 3} \int_{\mathbb{R}^{3}}\left(\sigma+n_{\infty}\right)\left|\partial^{\alpha} c\right|^{2} d x \leq C \int_{\mathbb{R}^{3}}|\nabla(\sigma, c, u)|^{2} d x,
\end{aligned}
$$

for some positive constants $C_{\alpha}$ and $\lambda$. Therefore (2.11) follows from the further linear combination of (2.16) and (2.20) and the time integration over $[0, T]$. This completes the proof of Proposition 2.1.

\subsection{Proof of local and global existence}

This subsection is devoted to the proof of Theorem 2.1. We construct the solution sequence $\left(n^{j}, c^{j}, u^{j}\right)_{j \geq 0}$ by solving iteratively the Cauchy problems on the following linear equations

$$
\left\{\begin{array}{l}
\partial_{t} n^{j+1}+u^{j} \cdot \nabla n^{j+1}=\delta \Delta n^{j+1}-\nabla \cdot\left(\chi\left(c^{j}\right) n^{j} \nabla c^{j+1}\right), \\
\partial_{t} c^{j+1}+u^{j} \cdot \nabla c^{j+1}=\mu \Delta c^{j+1}-k^{\prime}(0) n^{j} c^{j+1}-\left[k\left(c^{j}\right)-k^{\prime}(0) c^{j}\right] n^{j+1}, \\
\partial_{t} u^{j+1}+u^{j} \cdot \nabla u^{j+1}+\nabla P^{j+1}=\nu \Delta u^{j+1}-n^{j} \nabla \phi \\
\nabla \cdot u^{j+1}=0, \quad t>0, x \in \mathbb{R}^{3}
\end{array}\right.
$$

with

$$
\left.\left(n^{j+1}, c^{j+1}, u^{j+1}\right)\right|_{t=0}=\left(n_{0}, c_{0}, u_{0}\right), \quad x \in \mathbb{R}^{3},
$$

for $j \geq 0$, where $\left(n^{0}, c^{0}, u^{0}\right) \equiv\left(n_{\infty}, 0,0\right)$ is set at initial step. In terms of the perturbation $\sigma^{j}=$ $n^{j}-n_{\infty}$, the above Cauchy problems are reformulated as

$$
\left\{\begin{aligned}
& \partial_{t} \sigma^{j+1}-\delta \Delta \sigma^{j+1}+n_{\infty} \chi(0) \Delta c^{j+1}=-u^{j} \cdot \nabla \sigma^{j+1}-\nabla \cdot\left(\chi\left(c^{j}\right) \sigma^{j} \nabla c^{j+1}\right) \\
&-n_{\infty} \nabla \cdot\left(\left[\chi\left(c^{j}\right)-\chi(0)\right] \nabla c^{j+1}\right), \\
& \partial_{t} c^{j+1}-\mu \Delta c^{j+1}+n_{\infty} k^{\prime}(0) c^{j+1}=-u^{j} \cdot \nabla c^{j+1}-k^{\prime}(0) \sigma^{j} c^{j+1} \\
&-\left[k\left(c^{j}\right)-k^{\prime}(0) c^{j}\right]\left(n_{\infty}+\sigma^{j+1}\right), \\
& \partial_{t} u^{j+1}+\nabla P^{j+1}-\nu \Delta u^{j+1}=-u^{j} \cdot \nabla u^{j+1}-\sigma^{j} \nabla \phi, \\
& \nabla \cdot u^{j+1}=0, \quad t>0, x \in \mathbb{R}^{3},
\end{aligned}\right.
$$

with

$$
\left.\left(\sigma^{j+1}, c^{j+1}, u^{j+1}\right)\right|_{t=0}=\left(\sigma_{0}, c_{0}, u_{0}\right), \quad x \in \mathbb{R}^{3},
$$

for $j \geq 0$, where $\left(\sigma^{0}, c^{0}, u^{0}\right) \equiv(0,0,0)$ holds. In what follows, let us write $W^{j}=\left(n^{j}, c^{j}, u^{j}\right)$ and $W_{0}=\left(\sigma_{0}, c_{0}, u_{0}\right)$ for simplicity. Then, one has

Lemma 2.2. Let all conditions in Theorem 2.1 hold. There are constants $\epsilon_{1}>0, T_{1}>0, M_{1}>0$ such that if $\left\|W_{0}\right\|_{H^{3}} \leq \epsilon_{1}$, then for each $j \geq 0, W^{j} \in C\left(\left[0, T_{1}\right] ; H^{3}\right)$ is well-defined and

$$
\sup _{0 \leq t \leq T_{1}}\left\|W^{j}(t)\right\|_{H^{3}} \leq M_{1}, \quad j \geq 0 .
$$


Furthermore, $\left(W^{j}\right)_{j \geq 0}$ is a Cauchy sequence in the Banach space $C\left(\left[0, T_{1}\right] ; H^{3}\right)$, the corresponding limit function denoted by $W$ belongs to $C\left(\left[0, T_{1}\right] ; H^{3}\right)$ with

$$
\sup _{0 \leq t \leq T_{1}}\|W(t)\|_{H^{3}} \leq M_{1},
$$

and $W=(\sigma, c, u)$ is a solution over $\left[0, T_{1}\right]$ to the Cauchy problem (2.3)-(2.4). Finally, the Cauchy problem (2.3)-(2.4) admits at most one solution in $C\left(\left[0, T_{1}\right] ; H^{3}\right)$ satisfying $(2.23)$.

Proof. First of all, we prove (2.22) by induction. The trivial case is $j=0$ since $W^{0}=0$ by the assumption at initial step. Suppose that it is true for $j \geq 0$ with $M_{1}>0$ small enough to be determined later. To prove it for $j+1$, we need some energy estimates on $W^{j+1}$. From $(2.21)_{1}$, it holds for any $|\alpha| \leq 3$ that

$$
\begin{aligned}
& \frac{1}{2} \frac{d}{d t}\left\|\partial^{\alpha} \sigma^{j+1}\right\|^{2}+\delta\left\|\nabla \partial^{\alpha} \sigma^{j+1}\right\|^{2} \\
& =-\int_{\mathbb{R}^{3}} \partial^{\alpha}\left(u^{j} \cdot \nabla \sigma^{j+1}\right) \partial^{\alpha} \sigma^{j+1} d x+n_{\infty} \chi(0) \int_{\mathbb{R}^{3}} \nabla \partial^{\alpha} c^{j+1} \cdot \nabla \partial^{\alpha} \sigma^{j+1} d x \\
& \quad+\int_{\mathbb{R}^{3}} \partial^{\alpha}\left[\chi\left(c^{j}\right) \sigma^{j} \nabla c^{j+1}\right] \nabla \partial^{\alpha} \sigma^{j+1} d x+n_{\infty} \int_{\mathbb{R}^{3}} \partial^{\alpha}\left[\left(\chi\left(c^{j}\right)-\chi(0)\right) \nabla c^{j+1}\right] \nabla \partial^{\alpha} \sigma^{j+1} d x,
\end{aligned}
$$

where the r.h.s. is further bounded by

$$
\begin{gathered}
C\left\|u^{j}\right\|_{H^{3}}\left\|\nabla \sigma^{j+1}\right\|_{H^{3}}^{2}+C\left\|\nabla \partial^{\alpha} c^{j+1}\right\|\left\|\nabla \partial^{\alpha} \sigma^{j+1}\right\| \\
+C\left\|\sigma^{j}\right\|_{H^{3}}\left\|\nabla c^{j+1}\right\|_{H^{3}}\left\|\nabla \sigma^{j+1}\right\|_{H^{3}}+C\left\|c^{j}\right\|_{H^{3}}\left\|\nabla c^{j+1}\right\|_{H^{3}}\left\|\nabla \sigma^{j+1}\right\|_{H^{3}} .
\end{gathered}
$$

Then, after taking summation over $|\alpha| \leq 3$ and using the Cauchy inequality, one has

$$
\begin{gathered}
\frac{1}{2} \frac{d}{d t}\left\|\sigma^{j+1}\right\|_{H^{3}}^{2}+\lambda\left\|\nabla \sigma^{j+1}\right\|_{H^{3}}^{2} \\
\leq C\left\|\nabla c^{j+1}\right\|_{H^{3}}^{2}+C\left\|\left(\sigma^{j}, c^{j}, u^{j}\right)\right\|_{H^{3}}^{2}\left\|\nabla\left(\sigma^{j+1}, c^{j+1}\right)\right\|_{H^{3}}^{2} .
\end{gathered}
$$

Similarly from $(2.21)_{2}$ and $(2.21)_{3}$, one has

$$
\begin{gathered}
\frac{1}{2} \frac{d}{d t}\left\|c^{j+1}\right\|_{H^{3}}^{2}+\lambda\left\|\nabla c^{j+1}\right\|_{H^{3}}^{2} \\
\leq C\left\|c^{j}\right\|_{H^{3}}^{2}+C\left\|\left(\sigma^{j}, c^{j}, u^{j}\right)\right\|_{H^{3}}^{2}\left\|\nabla\left(\sigma^{j+1}, c^{j+1}\right)\right\|_{H^{3}}^{2},
\end{gathered}
$$

and

$$
\frac{1}{2} \frac{d}{d t}\left\|u^{j+1}\right\|_{H^{3}}^{2}+\lambda\left\|\nabla u^{j+1}\right\|_{H^{3}}^{2} \leq C\left\|\sigma^{j}\right\|_{H^{3}}^{2}+C\left\|u^{j}\right\|_{H^{3}}^{2}\left\|\nabla u^{j+1}\right\|_{H^{3}}^{2} .
$$

Taking the linear combination of inequalities (2.24), (2.25) and (2.26), one has

$$
\begin{aligned}
& \frac{1}{2} \frac{d}{d t}\left(\left\|\sigma^{j+1}\right\|_{H^{3}}^{2}+d\left\|c^{j+1}\right\|_{H^{3}}^{2}+\left\|u^{j+1}\right\|_{H^{3}}^{2}\right)+\lambda\left\|\nabla\left(\sigma^{j+1}, c^{j+1}, u^{j+1}\right)\right\|_{H^{3}}^{2} \\
& \quad \leq C\left\|\left(\sigma^{j}, c^{j}\right)\right\|_{H^{3}}^{2}+C\left\|\left(\sigma^{j}, c^{j}, u^{j}\right)\right\|_{H^{3}}^{2}\left\|\nabla\left(\sigma^{j+1}, c^{j+1}, u^{j+1}\right)\right\|_{H^{3}}^{2},
\end{aligned}
$$

for some constant $d>0$ large enough. Further, after taking time integration, it holds that

$$
\begin{gathered}
\left\|W^{j+1}(t)\right\|_{H^{3}}^{2}+\lambda \int_{0}^{t}\left\|\nabla W^{j+1}(s)\right\|_{H^{3}}^{2} d s \leq C\left\|W_{0}\right\|_{H^{3}}^{2}+C \int_{0}^{t}\left\|W^{j}(s)\right\|_{H^{3}}^{2} d s \\
+C \int_{0}^{t}\left\|W^{j}(s)\right\|_{H^{3}}^{2}\left\|\nabla W^{j+1}(s)\right\|_{H^{3}}^{2} d s,
\end{gathered}
$$


which from the inductive assumption implies

$$
\left\|W^{j+1}(t)\right\|_{H^{3}}^{2}+\lambda \int_{0}^{t}\left\|\nabla W^{j+1}(s)\right\|_{H^{3}}^{2} d s \leq C \epsilon_{1}^{2}+C M_{1}^{2} T_{1}+C M_{1}^{2} \int_{0}^{t}\left\|\nabla W^{j+1}(s)\right\|_{H^{3}}^{2} d s,
$$

for any $0 \leq t \leq T_{1}$. Now, one can take properly small constants $\epsilon_{1}>0, T_{1}>0$ and $M_{1}>0$ such that

$$
\left\|W^{j+1}(t)\right\|_{H^{3}}^{2}+\lambda \int_{0}^{t}\left\|\nabla W^{j+1}(s)\right\|_{H^{3}}^{2} d s \leq M_{1}^{2},
$$

for any $0 \leq t \leq T_{1}$. This implies that (2.22) holds true for $j+1$ if so for $j$. Hence (2.22) is proved for all $j \geq 0$.

Next, define the equivalent energy functional $\mathcal{E}\left(W^{j+1}(t)\right)$ by

$$
\mathcal{E}\left(W^{j+1}(t)\right)=\left\|\sigma^{j+1}\right\|_{H^{3}}^{2}+d\left\|c^{j+1}\right\|_{H^{3}}^{2}+\left\|u^{j+1}\right\|_{H^{3}}^{2},
$$

where the constant $d>0$ is given in (2.27). Similar to prove (2.27), one has

$$
\begin{aligned}
& \left|\mathcal{E}\left(W^{j+1}(t)\right)-\mathcal{E}\left(W^{j+1}(s)\right)\right|=\left|\int_{s}^{t} \frac{d}{d \theta} \mathcal{E}\left(W^{j+1}(\theta)\right) d \theta\right| \\
& \leq C \int_{s}^{t}\left\|W^{j}(\theta)\right\|_{H^{3}}^{2} d \theta+C \int_{s}^{t}\left(1+\left\|W^{j}(\theta)\right\|_{H^{3}}^{2}\right)\left\|\nabla W^{j}(\theta)\right\|_{H^{3}}^{2} d \theta \\
& \leq C M_{1}^{2}(t-s)+C\left(1+M_{1}^{2}\right) \int_{s}^{t}\left\|\nabla W^{j}(\theta)\right\|_{H^{3}}^{2} d \theta,
\end{aligned}
$$

for any $0 \leq s \leq t \leq T_{1}$. Here, the time integral in the second term on the r.h.s. is finite due to (2.28), and hence $\mathcal{E}\left(W^{j+1}(t)\right)$ is continuous in $t$ for each $j \geq 0$. On the other hand, from (2.25) and (2.26), the same argument can be applied to $c^{j+1}$ and $u^{j+1}$ so that both $\left\|c^{j+1}(t)\right\|^{2}$ and $\left\|u^{j+1}(t)\right\|^{2}$ are continuous in $t$, and so is $\left\|\sigma^{j+1}(t)\right\|^{2}$. Therefore, $\left\|W^{j}(t)\right\|_{H^{3}}^{2}$ is continuous in time for each $j \geq 1$.

In order to consider the convergence of the sequence $\left(W^{j}\right)_{j \geq 0}$, let us take the difference of $(2.21)$ for $j$ and $j-1$ so that it gives

$$
\left\{\begin{array}{l}
\partial_{t}\left(\sigma^{j+1}-\sigma^{j}\right)-\delta \Delta\left(\sigma^{j+1}-\sigma^{j}\right)+n_{\infty} \chi(0) \Delta\left(c^{j+1}-c^{j}\right) \\
=-u^{j} \cdot \nabla\left(\sigma^{j+1}-\sigma^{j}\right)-\left(u^{j}-u^{j-1}\right) \cdot \nabla \sigma^{j} \\
\quad-\nabla \cdot\left[\chi\left(c^{j}\right) \sigma^{j} \nabla\left(c^{j+1}-c^{j}\right)\right]-\nabla \cdot\left[\left(\chi\left(c^{j}\right) \sigma^{j}-\chi\left(c^{j-1}\right) \sigma^{j-1}\right) \nabla c^{j}\right] \\
\quad-n_{\infty} \nabla \cdot\left(\left[\chi\left(c^{j}\right)-\chi(0)\right] \nabla\left(c^{j+1}-c^{j}\right)\right)-n_{\infty} \nabla \cdot\left(\left[\chi\left(c^{j}\right)-\chi\left(c^{j-1}\right)\right] \nabla c^{j}\right), \\
\partial_{t}\left(c^{j+1}-c^{j}\right)-\mu \Delta\left(c^{j+1}-c^{j}\right)+n_{\infty} k^{\prime}(0)\left(c^{j+1}-c^{j}\right) \\
=-u^{j} \cdot \nabla\left(c^{j+1}-c^{j}\right)-\left(u^{j}-u^{j-1}\right) \cdot \nabla c^{j}-k^{\prime}(0) \sigma^{j}\left(c^{j+1}-c^{j}\right)-k^{\prime}(0)\left(\sigma^{j}-\sigma^{j-1}\right) c^{j} \\
\quad-\int_{0}^{c^{j}} \int_{0}^{\eta} k^{\prime \prime}(\theta) d \theta d \eta\left(\sigma^{j+1}-\sigma^{j}\right)-\int_{c^{j-1}}^{c^{j}} \int_{0}^{\eta} k^{\prime \prime}(\theta) d \theta d \eta \sigma^{j}, \\
\partial_{t}\left(u^{j+1}-u^{j}\right)+\nabla P^{j+1, j}-\nu \Delta\left(u^{j+1}-u^{j}\right) \\
=-u^{j} \cdot \nabla\left(u^{j+1}-u^{j}\right)-\left(u^{j}-u^{j-1}\right) \cdot \nabla u^{j}-\left(\sigma^{j}-\sigma^{j-1}\right) \nabla \phi, \\
\nabla \cdot\left(u^{j+1}-u^{j}\right)=0, \quad t>0, x \in \mathbb{R}^{3} .
\end{array}\right.
$$

By using the same energy estimates as before, one has

$$
\begin{gathered}
\frac{1}{2} \frac{d}{d t} \mathcal{E}\left(W^{j+1}-W^{j}\right)+\lambda\left\|\nabla\left(W^{j+1}-W^{j}\right)\right\|_{H^{3}}^{2} \leq C\left\|W^{j}-W^{j-1}\right\|_{H^{3}}^{2} \\
+C\left\|W^{j}-W^{j-1}\right\|_{H^{3}}^{2}\left\|\nabla\left(W^{j}, W^{j-1}\right)\right\|_{H^{3}}^{2}+C\left\|\left(W^{j}, W^{j-1}\right)\right\|_{H^{3}}^{2}\left\|\nabla\left(W^{j+1}-W^{j}\right)\right\|_{H^{3}}^{2} .
\end{gathered}
$$


The further time integration gives

$$
\begin{gathered}
\left\|W^{j+1}(t)-W^{j}(t)\right\|_{H^{3}}^{2}+\lambda \int_{0}^{t}\left\|\nabla\left(W^{j+1}(s)-W^{j}(s)\right)\right\|_{H^{3}}^{2} d s \\
\leq C\left(1+M_{1}^{2}\right) T_{1} \sup _{0 \leq s \leq T_{1}}\left\|W^{j}(s)-W^{j-1}(s)\right\|_{H^{3}}^{2}+C M_{1}^{2} \int_{0}^{t}\left\|\nabla\left(W^{j+1}(s)-W^{j}(s)\right)\right\|_{H^{3}}^{2} d s,
\end{gathered}
$$

for any $0 \leq t \leq T_{1}$, which by smallness of $T_{1}$ and $M_{1}$ implies that there is a constant $\lambda<1$ such that

$$
\sup _{0 \leq t \leq T_{1}}\left\|W^{j+1}(t)-W^{j}(t)\right\|_{H^{3}} \leq \lambda \sup _{0 \leq t \leq T_{1}}\left\|W^{j}(t)-W^{j-1}(t)\right\|_{H^{3}},
$$

for any $j \geq 1$. It can be seen from the above inequality that $\left(W^{j}\right)_{j \geq 0}$ is a Cauchy sequence in the Banach space $C\left(\left[0, T_{1}\right] ; H^{3}\right)$. Thus the limit function

$$
W=W^{0}+\lim _{m \rightarrow \infty} \sum_{j=0}^{m}\left(W^{j+1}-W^{j}\right)
$$

indeed exists in $C\left(\left[0, T_{1}\right] ; H^{3}\right)$, and satisfies

$$
\sup _{0 \leq t \leq T_{1}}\|W(t)\|_{H^{3}} \leq \sup _{0 \leq t \leq T_{1}} \liminf _{j \rightarrow \infty}\left\|W^{j}(t)\right\|_{H^{3}} \leq M_{1} .
$$

Finally, suppose that $W$ and $\widetilde{W}$ are two solutions in $C\left(\left[0, T_{1}\right] ; H^{3}\right)$ satisfying (2.23). By applying the same process as in (2.29) to prove the convergence of $\left(W^{j}\right)_{j \geq 0}$, one has

$$
\sup _{0 \leq t \leq T_{1}}\|W(t)-\widetilde{W}(t)\|_{H^{3}} \leq \lambda \sup _{0 \leq t \leq T_{1}}\|W(t)-\widetilde{W}(t)\|_{H^{3}}
$$

for the constant $\lambda<1$. Hence, $W=\widetilde{W}$ holds. This proves the uniqueness and thus completes the proof of Lemma 2.2 .

Proof of Theorem 2.1: Choose a positive constant

$$
M=\min \left\{\epsilon_{0}, \epsilon_{1}\right\},
$$

where $\epsilon_{0}>0$ and $\epsilon_{1}>0$ are given in Lemma 2.1 and Lemma 2.2, respectively. Further choose initial data $W_{0}=\left(\sigma_{0}, c_{0}, u_{0}\right)$ such that

$$
\left\|W_{0}\right\|_{H^{3}} \leq \frac{M}{2 \sqrt{1+C_{0}}}
$$

where $C_{0}>0$ is given in Lemma 2.1. Define the lifespan of solutions to the Cauchy problem (2.3)-(2.4) by

$$
T=\sup \left\{t ; \sup _{0 \leq s \leq t}\|W(s)\|_{H^{3}} \leq M\right\}
$$

Since

$$
\left\|W_{0}\right\|_{H^{3}} \leq \frac{M}{2 \sqrt{1+C_{0}}} \leq \frac{M}{2}<M \leq \epsilon_{1},
$$

then $T>0$ holds true from the local existence result Lemma 2.2 and the continuation argument. If $T$ is finite, it follows from the definition of $T$ that

$$
\sup _{0 \leq s \leq T}\|W(s)\|_{H^{3}}=M
$$

which is a contradiction to the fact from uniform a priori estimates that

$$
\sup _{0 \leq s \leq T}\|W(s)\|_{H^{3}} \leq \sqrt{C_{0}}\left\|W_{0}\right\| \leq \frac{M \sqrt{C_{0}}}{2 \sqrt{1+C_{0}}} \leq \frac{M}{2} .
$$


Therefore, $T=\infty$. This implies that the local solution $W(t)$ obtained in Lemma 2.2 can be extent to infinite time. Thus, the Cauchy problem $(2.3)-(2.4)$ admits a solution $W=(\sigma, c, u)$ in $C\left([0, \infty) ; H^{3}\right)$ with $\sigma+n_{\infty} \geq 0$ and $c \geq 0$, and uniqueness of solutions also follows from Lemma 2.2. Finally, (2.6) follows from (2.11). This completes the proof of Theorem 2.1.

\subsection{Proof of rates of convergence}

Throughout this subsection, we suppose that $n_{\infty}=0$, all conditions in Theorem 2.1 hold and also

$$
\begin{aligned}
\|(\sigma, c, u)(t)\|_{H^{3}}^{2} & +\lambda \int_{0}^{t} \int_{\mathbb{R}^{3}} \sigma\left[k(c) c+k^{\prime}(0) \sum_{1 \leq|\alpha| \leq 3}\left|\partial^{\alpha} c(s)\right|^{2}\right] d x d s \\
& +\lambda \int_{0}^{t}\|\nabla(\sigma, c, u)(s)\|_{H^{3}}^{2} d s \leq C \epsilon
\end{aligned}
$$

for any $t \geq 0$, where $\epsilon>0$ is a small constant. For later use, let us define the rate index $\gamma_{p, q}$ by

$$
\gamma_{p, q}=\frac{3}{2}\left(\frac{1}{q}-\frac{1}{p}\right)
$$

for any $1 \leq q \leq p \leq \infty$.

Proof of Theorem 2.2: It is divided into three steps corresponding to the estimates of the timedecay rates for $c, \sigma$ and $u$, respectively.

Time-decay of $c$. When $n_{\infty}=0,(2.3)_{2}$ also reads

$$
\partial_{t} c+u \cdot \nabla c-\mu \Delta c+k(c) \sigma=0 .
$$

Take $2 \leq p<\infty$. Multiplying the above equation by $p c^{p-1}$ and then integrating, one has

$$
\frac{d}{d t} \int_{\mathbb{R}^{3}} c^{p} d x+\frac{4 \mu(p-1)}{p} \int_{\mathbb{R}^{3}}\left|\nabla c^{p / 2}\right|^{2} d x \leq 0 .
$$

Notice that since $k(c) \geq 0$ and $\sigma \geq 0,(2.30)$ also gives

$$
\|c(t)\|_{L^{1}} \leq\left\|c_{0}\right\|_{L^{1}}
$$

for any $t \geq 0$. Thus (2.8) can be proved by using the method in [20]. Here, for completeness, we give the proof of (2.8). Let $\alpha>0$. Multiplying $(2.31)$ by $(1+t)^{\alpha}$ and taking integration over $[0, t]$, one has

$$
\begin{aligned}
& (1+t)^{\alpha}\|c(t)\|_{L^{p}}^{p}+\frac{4 \mu(p-1)}{p} \int_{0}^{t}(1+s)^{\alpha}\left\|\nabla c^{p / 2}(s)\right\|^{2} d s \\
& \leq\left\|c_{0}\right\|_{L^{p}}^{p}+\alpha \int_{0}^{t}(1+s)^{\alpha-1}\|c(s)\|_{L^{p}}^{p} d s .
\end{aligned}
$$

Recalling the interpolation inequality

$$
\|f\|_{L^{p}} \leq C\left\|\nabla|f|^{p / 2}\right\|^{\frac{2 \gamma_{p, q}}{1+p \gamma_{p}, q}}\|f\|_{L^{q}}^{\frac{1}{1+p \gamma_{p}, q}}
$$

for any $2 \leq p<\infty$ and $1 \leq q \leq p$, one has

$$
\begin{aligned}
& \alpha \int_{0}^{t}(1+s)^{\alpha-1}\|c(s)\|_{L^{p}}^{p} d s \\
& \leq C \int_{0}^{t}(1+t)^{\alpha-1}\left\|\nabla c^{p / 2}\right\|^{2 p \gamma_{p, 1} /\left(1+p \gamma_{p, q}\right)}\|c(s)\|_{L^{1}}^{p /\left(1+p \gamma_{p, 1}\right)} d s \\
& \leq \eta \int_{0}^{t}(1+s)^{\alpha}\left\|\nabla c^{p / 2}(s)\right\|^{2} d s+\frac{C}{\eta} \int_{0}^{t}(1+s)^{\alpha-p \gamma_{p, 1}-1}\|c(s)\|_{L^{1}}^{p} d s \\
& \leq \eta \int_{0}^{t}(1+s)^{\alpha}\left\|\nabla c^{p / 2}(s)\right\|^{2} d s+\frac{C}{\eta}\left\|c_{0}\right\|_{L^{1}}^{p}(1+t)^{\alpha-p \gamma_{p, 1}},
\end{aligned}
$$


for any $\eta>0$, where the Young inequality $p \gamma_{p, 1} /\left(1+p \gamma_{p, 1}\right)+1 /\left(1+p \gamma_{p, 1}\right)=1$ and $(2.32)$ were used and $\alpha>p \gamma_{p, 1}$ was supposed. Therefore, (2.8) for $2 \leq p<\infty$ follows from (2.33) and (2.34) by taking $\eta>0$ small enough. (2.8) for $1 \leq p<2$ follows from the interpolation inequality

$$
\|c\|_{L^{p}} \leq\|c\|_{L^{1}}^{2 / p-1}\|c\|^{2(p-1) / p} .
$$

Time-decay of $\sigma$. Recall the equation of $\sigma$ when $n_{\infty}=0$ :

$$
\partial_{t} \sigma+u \cdot \sigma-\delta \Delta \sigma=-\nabla \cdot(\chi(c) \sigma \nabla c) .
$$

Similar as in $(2.31)$, for $2 \leq p<\infty$, one has

$$
\begin{aligned}
\frac{d}{d t}\|\sigma(t)\|_{L^{p}}^{p}+\frac{4 \delta(p-1)}{p}\left\|\nabla \sigma^{p / 2}(t)\right\|^{2} & \leq p(p-1) \int_{\mathbb{R}^{3}} \chi(c) \nabla c \cdot \sigma^{p-1} \nabla \sigma d x \\
& \leq C\|\nabla c\|_{L^{3}}\left\|\sigma^{p / 2}\right\|_{L^{6}}\left\|\sigma^{p / 2-1} \nabla \sigma\right\| \\
& \leq C\|\nabla c\|_{H^{1}}\left\|\nabla \sigma^{p / 2}\right\|^{2} \\
& \leq C \epsilon\left\|\nabla \sigma^{p / 2}\right\|^{2}
\end{aligned}
$$

which implies by the smallness of $\epsilon$ that

$$
\frac{d}{d t}\|\sigma(t)\|_{L^{p}}^{p}+\frac{4 \delta(p-1)}{p}\left\|\nabla \sigma^{p / 2}(t)\right\|^{2} \leq 0 .
$$

Since $\|\sigma(t)\|_{L^{1}} \leq\left\|\sigma_{0}\right\|_{L^{1}}$ for any $t \geq 0,(2.7)$ for any $1 \leq p<\infty$ similarly follows.

Time-decay of $u$. In order to obtain the time-decay rate of $u$, we first consider the time-decay of high-order derivatives of $(\sigma, c)$. The high-order energy estimates (2.17) and (2.18) give

$$
\frac{d}{d t}\|\nabla(\sigma, c)\|_{H^{2}}^{2}+\lambda\|\nabla(\sigma, c)\|_{H^{3}}^{2} \leq C\|\nabla(\sigma, c)\|^{2} .
$$

One can use the spectral analysis of the classical heat operator to get the time-decay of $\|\nabla(\sigma, c)\|^{2}$. For this purpose, one can write $\sigma, c$ as

$$
\begin{aligned}
& \sigma(t)=e^{\delta \Delta t} \sigma_{0}+\int_{0}^{t} e^{\delta \Delta(t-s)}(-u \cdot \nabla \sigma-\nabla \cdot(\chi(c) \sigma \nabla c)) d s, \\
& c(t)=e^{\mu \Delta t} c_{0}+\int_{0}^{t} e^{\mu \Delta(t-s)}(-u \cdot \nabla c-k(c) \sigma) d s .
\end{aligned}
$$

Recall

$$
\left\|\partial^{m} e^{\lambda \Delta t} f\right\| \leq C\|f\|_{L^{p} \cap H^{m}}(1+t)^{-\gamma_{2, p}-m / 2},
$$

for $1 \leq p \leq 2$ and integer $m \geq 0$. Then, for $1 \leq p \leq 2$, applying (2.38) to (2.36) and (2.37), one has

$$
\begin{aligned}
\|\nabla \sigma(t)\| \leq & C\left\|\sigma_{0}\right\|_{L^{p} \cap H^{1}}(1+t)^{-\gamma_{2, p}-1 / 2} \\
& +C \int_{0}^{t}(1+t-s)^{-5 / 4}\left(\|u \cdot \nabla \sigma\|_{L^{1} \cap H^{1}}+\|\chi(c) \sigma \nabla c\|_{L^{1} \cap H^{2}}\right) d s \\
\leq & C\left\|\sigma_{0}\right\|_{L^{p} \cap H^{1}}(1+t)^{-\gamma_{2, p}-1 / 2} \\
& +C \epsilon \int_{0}^{t}(1+t-s)^{-5 / 4}\|\nabla(\sigma, c)(s)\|_{H^{1}} d s,
\end{aligned}
$$


and

$$
\begin{aligned}
\|\nabla c(t)\| \leq & C\left\|c_{0}\right\|_{L^{p} \cap H^{1}}(1+t)^{-\gamma_{2, p}-1 / 2} \\
& +C \int_{0}^{t}(1+t-s)^{-5 / 4}\left(\|u \cdot \nabla c\|_{L^{1} \cap H^{1}}+\|k(c) \sigma\|_{L^{1} \cap H^{1}}\right) d s \\
\leq & C\left\|c_{0}\right\|_{L^{1} \cap H^{1}}(1+t)^{-\gamma_{2, p}-1 / 2}+C \epsilon \int_{0}^{t}(1+t-s)^{-5 / 4}\|\nabla c(s)\|_{H^{1}} d s \\
& +C(1+t)^{-5 / 4}\left\|c_{0}\right\|_{L^{1} \cap L^{2}}\left\|\sigma_{0}\right\|_{L^{1} \cap L^{2}} .
\end{aligned}
$$

Define the high-order energy $\mathcal{E}(t)$ and its weighted norm $\mathcal{E}_{\infty}(t)$ by

$$
\mathcal{E}(t)=\|\nabla(\sigma, c)(t)\|_{H^{2}}^{2}, \quad \mathcal{E}_{\infty}(t)=\sup _{0 \leq s \leq t}(1+s)^{2 \gamma_{2, p}+1} \mathcal{E}(s) .
$$

Then, (2.39) and (2.40) implies

$$
\|\nabla(\sigma, c)(t)\| \leq C(1+t)^{-\gamma_{2, p}-1 / 2}\left(K_{p}+\epsilon \sqrt{\mathcal{E}_{\infty}(t)}\right),
$$

where $K_{p}$ is denoted by

$$
K_{p}=\left\|\left(\sigma_{0}, c_{0}\right)\right\|_{L^{p} \cap H^{1}}+\left\|\sigma_{0}\right\|_{L^{1} \cap L^{2}}\left\|c_{0}\right\|_{L^{1} \cap L^{2}} .
$$

Therefore, by the Gronwall inequality, it follows from (2.35) that

$$
\begin{aligned}
\mathcal{E}(t) & \leq \mathcal{E}(0) e^{-\lambda t}+C \int_{0}^{t} e^{-\lambda(t-s)}\|\nabla(\sigma, c)(s)\|^{2} d s \\
& \leq C\left(\mathcal{E}(0)+K_{p}^{2}+\epsilon \mathcal{E}_{\infty}(t)\right)(1+t)^{-2 \gamma_{2, p}-1}
\end{aligned}
$$

which implies

$$
\mathcal{E}_{\infty}(t) \leq C\left(\mathcal{E}(0)+K_{p}^{2}+\epsilon \mathcal{E}_{\infty}(t)\right)
$$

for any $t \geq 0$. Since $\epsilon>0$ is small enough, $\mathcal{E}_{\infty}(t)$ is uniformly bounded in time and hence, from the definition of $\mathcal{E}_{\infty}(t)$, it holds that

$$
\|\nabla(\sigma, c)(t)\|_{H^{2}} \leq C\left(\left\|\nabla\left(\sigma_{0}, c_{0}\right)\right\|_{H^{2}}+K_{p}\right)(1+t)^{-\gamma_{2, p}-1 / 2},
$$

for any $1 \leq p \leq 2$ and any $t \geq 0$.

Next, we use the same method to deal with the time-decay of high-order derivatives of $u$. Define the projection operator $\mathbf{P}$ by

$$
\mathbf{P}=\mathbf{I}-\nabla \Delta^{-1} \nabla \cdot
$$

where $\mathbf{I}$ is the identity. Applying $\mathbf{P}$ to $(2.3)_{3}$ yields

$$
\partial_{t} u-\nu \Delta u=-\mathbf{P}(u \cdot \nabla u)-\mathbf{P}(\sigma \nabla \phi) .
$$

Notice that $\mathbf{P} \nabla(\sigma \phi)=0$, and hence

$$
\mathbf{P}(\sigma \nabla \phi)=-\mathbf{P}(\phi \nabla \sigma) .
$$

(2.42) can be written as

$$
u(t)=e^{\nu \Delta t} u_{0}+\int_{0}^{t} e^{\nu \Delta(t-s)}(-\mathbf{P}(u \cdot \nabla u)+\mathbf{P}(\phi \nabla \sigma)) d s .
$$

Let $1<p \leq 2$ and $1<q \leq p$ with $q$ close to 1 . Then, it holds that

$$
\begin{gathered}
\|\nabla u(t)\| \leq C\left\|u_{0}\right\|_{L^{p} \cap H^{1}}(1+t)^{-\gamma_{2, p}-1 / 2} \\
+C \int_{0}^{t}(1+t-s)^{-\gamma_{2, q}-1 / 2}\left(\|\mathbf{P}(u \cdot \nabla u)\|_{L^{q} \cap H^{1}}+\|\mathbf{P}(\phi \nabla \sigma)\|_{L^{q} \cap H^{1}}\right) d s
\end{gathered}
$$


From the Riesz inequality, $\|\mathbf{P} f\|_{L^{r}} \leq C\|f\|_{L^{r}}$ holds for any $1<r<\infty$, and thus one has

$$
\|\mathbf{P}(u \cdot \nabla u)\|_{L^{q} \cap H^{1}}+\|\mathbf{P}(\nabla \sigma \phi)\|_{L^{q} \cap H^{1}} \leq C\|u \cdot \nabla u\|_{L^{q} \cap H^{1}}+C\|\phi \nabla \sigma\|_{L^{q} \cap H^{1}} .
$$

Here, for $H^{1}$-norm, it holds that

$$
\begin{aligned}
\|u \cdot \nabla u\|_{H^{1}} & \leq C \epsilon\|\nabla u\|_{H^{1}}, \\
\|\phi \nabla \sigma\|_{H^{1}} & \leq C\|\nabla \sigma\|_{H^{1}} \sup _{t}\|\phi(t)\|_{W^{1, \infty}},
\end{aligned}
$$

and for $L^{q}$-norm, it follows from the Hölder inequality that

$$
\begin{aligned}
\|u \cdot \nabla u\|_{L^{q}} & \leq\|\nabla u\| \cdot\|u\|_{L^{2 q /(2-q)}} \leq C\|\nabla u\| \cdot\|u\|_{H^{2}} \leq C \epsilon\|\nabla u\|, \\
\|\phi \nabla \sigma\|_{L^{q}} & \leq\|\nabla \sigma\| \sup _{t}\|\phi(t)\|_{L^{2 q /(2-q)}},
\end{aligned}
$$

which imply that

$$
\|\mathbf{P}(u \cdot \nabla u)\|_{L^{q} \cap H^{1}}+\|\mathbf{P}(\phi \nabla \sigma)\|_{L^{q} \cap H^{1}} \leq C \epsilon\|\nabla u\|_{H^{1}}+C_{\phi}\|\nabla \sigma\|_{H^{1}},
$$

where $C_{\phi}$ only depends on the $L^{\infty}\left(\mathbb{R}^{+} ; L^{2 q /(2-q)}\left(\mathbb{R}^{3}\right) \cap W^{1, \infty}\left(\mathbb{R}^{3}\right)\right)$-norm of $\phi$. Thus, (2.43) together with (2.41) and (2.44) give

$$
\begin{aligned}
\|\nabla u(t)\| \leq & C\left(\left\|u_{0}\right\|_{L^{p} \cap H^{1}}+K_{0}\right)(1+t)^{-\gamma_{2, p}-1 / 2} \\
& +C \epsilon \int_{0}^{t}(1+t-s)^{-\gamma_{2, q}-1 / 2}\|\nabla u(s)\|_{H^{1}} d s .
\end{aligned}
$$

From the high-order energy estimates, it holds that

$$
\frac{d}{d t}\|\nabla(\sigma, c, u)(t)\|_{H^{2}}^{2}+\lambda\|\nabla(\sigma, c, u)(t)\|_{H^{3}}^{2} \leq C\|\nabla(\sigma, c, u)\|^{2} .
$$

Therefore, similarly as before, by using (2.41), it follows from (2.45) and (2.46) that

$$
\|\nabla(\sigma, c, u)(t)\|_{H^{2}} \leq C\left(\left\|u_{0}\right\|_{L^{p} \cap H^{3}}+K_{0}\right)(1+t)^{-\gamma_{2, p}-1 / 2} .
$$

Then, similarly to (2.45), one has

$$
\begin{aligned}
\|u(t)\| \leq & C\left\|u_{0}\right\|_{L^{p} \cap L^{2}}(1+t)^{-\gamma_{2, p}} \\
& +C \int_{0}^{t}(1+t-s)^{-\gamma_{2, q}}\left(\epsilon\|\nabla u(s)\|+C_{\phi}\|\nabla \sigma(s)\|\right) d s .
\end{aligned}
$$

Since $1<p<6 / 5$ implies $\gamma_{2, p}+1 / 2>1$, then (2.9) follows from (2.47) and (2.48). This completes the proof of Theorem 2.2 .

We conclude this section with a remark on the time-decay rates of solutions when $n_{\infty}>0$ as mentioned in Remark 2.2. In fact, the linearized part of the system (2.3) reads

$$
\left\{\begin{array}{l}
\partial_{t} \sigma-\delta \Delta \sigma+n_{\infty} \Delta c=0 \\
\partial_{t} c-\mu \Delta c+n_{\infty} k^{\prime}(0) c=0 \\
\partial_{t} u+\nabla P-\nu \Delta u=-\sigma \nabla \phi \\
\nabla \cdot u=0
\end{array}\right.
$$

for $t \geq 0$ and $x \in \mathbb{R}^{3}$. Thus, if $k^{\prime}(0)>0$ holds, then $c$ decays exponentially in time, and otherwise it decays like a heat kernel. Moreover, the time-decay of $c$ in turn leads us to obtain the time-decay of $\sigma$ and then $u$ for which the decay rate in time is algebraic. 


\section{The coupled Chemotaxis-Stokes equations}

\subsection{Global weak solutions with large data}

In this section, we are concerned with the Cauchy problem on the coupled Chemotaxis-Stokes equations in $\Omega=\mathbb{R}^{2}$ :

$$
\left\{\begin{array}{l}
\partial_{t} n+u \cdot \nabla n=\delta \Delta n-\nabla \cdot(\chi(c) n \nabla c), \\
\partial_{t} c+u \cdot \nabla c=\mu \Delta c-k(c) n \\
\partial_{t} u+\nabla P=\nu \Delta u-n \nabla \phi \\
\nabla \cdot u=0, \quad t>0, x \in \mathbb{R}^{2}
\end{array}\right.
$$

with initial data

$$
\left.(n, c, u)\right|_{t=0}=\left(n_{0}(x), c_{0}(x), u_{0}(x)\right), \quad x \in \mathbb{R}^{2} .
$$

Notice that throughout this section, we shall suppose that the spatial domain is two dimensional and the study in the case of three space dimensions are left for future. Instead of the perturbation theory in the presence of convective terms developed in Section 2, in what follows we shall consider the nonperturbation existence theory for the above Cauchy problem when the initial cell density $n_{0}(x)$ has finite mass. Actually, under some conditions, the global existence of weak solutions can be established provided that the strength of the potential function $\phi$ is weak or the initial substrate concentration $c_{0}(x)$ is small in some sense.

First of all, let us give the definition of weak solutions.

Definition 3.1. $(n, c, u)$ is called a weak solution to the Cauchy problem (3.1)-(3.2) if the following two conditions hold:

(i) $n(t, x) \geq 0, c(t, x) \geq 0, t \geq 0, x \in \mathbb{R}^{2}$, and for any $T>0$,

$$
\left\{\begin{array}{l}
n(1+|x|+|\ln n|) \in L^{\infty}\left(0, T ; L^{1}\left(\mathbb{R}^{2}\right)\right), \nabla \sqrt{n} \in L^{2}\left(0, T ; L^{2}\left(\mathbb{R}^{2}\right)\right), \\
c \in L^{\infty}\left(0, T ; L^{1}\left(\mathbb{R}^{2}\right) \cap L^{\infty}\left(\mathbb{R}^{2}\right) \cap H^{1}\left(\mathbb{R}^{2}\right)\right), \nabla c \in L^{2}\left(0, T ; L^{2}\left(\mathbb{R}^{2}\right)\right), \\
\sqrt{n}|\nabla c| \in L^{2}\left(0, T ; L^{2}\left(\mathbb{R}^{2}\right)\right) \\
u \in L^{\infty}\left(0, T ; L^{2}\left(\mathbb{R}^{2}\right)\right), \nabla u \in L^{2}\left(0, T ; L^{2}\left(\mathbb{R}^{2}\right)\right)
\end{array}\right.
$$

(ii) $\forall \varphi \in C_{0}^{\infty}\left([0, \infty) \times \mathbb{R}^{2}\right)$,

$$
\begin{gathered}
\int_{0}^{\infty} \int_{\mathbb{R}^{2}} n\left(\partial_{t} \varphi+\delta \Delta \varphi\right) d x d t+\int_{0}^{\infty} \int_{\mathbb{R}^{2}} n u \cdot \nabla \varphi d x d t \\
+\int_{0}^{\infty} \int_{\mathbb{R}^{2}} \chi(c) n \nabla c \cdot \nabla \varphi d x d t+\int_{\mathbb{R}^{2}} n_{0}(x) \varphi(0, x) d x=0 \\
\int_{0}^{\infty} \int_{\mathbb{R}^{2}} c\left(\partial_{t} \varphi+\mu \Delta \varphi\right) d x d t+\int_{0}^{\infty} \int_{\mathbb{R}^{2}} c u \cdot \nabla \varphi d x d t \\
-\int_{0}^{\infty} \int_{\mathbb{R}^{2}} k(c) n \varphi d x d t+\int_{\mathbb{R}^{2}} c_{0}(x) \varphi(0, x) d x=0,
\end{gathered}
$$

and $\forall \widetilde{\varphi} \in C_{0}^{\infty}\left([0, \infty) \times \mathbb{R}^{2}\right)$ with $\nabla \cdot \widetilde{\varphi}=0$,

$$
\int_{0}^{\infty} \int_{\mathbb{R}^{2}} u \cdot\left(\partial_{t} \widetilde{\varphi}+\nu \Delta \widetilde{\varphi}\right) d x d t-\int_{0}^{\infty} \int_{\mathbb{R}^{2}} n \nabla \phi \cdot \widetilde{\varphi} d x d t+\int_{\mathbb{R}^{2}} u_{0}(x) \cdot \varphi(0, x) d x=0 .
$$

Next, to state the global existence results, the following assumptions are needed:

(B1) $\chi(c)>0, \chi^{\prime}(c) \geq 0, k^{\prime}(c)>0, \frac{d^{2}}{d c^{2}}\left(\frac{k(c)}{\chi(c)}\right)<0$; 
(B2)

$\phi=\phi(x) \geq 0, x \in \mathbb{R}^{2}, \nabla \phi \in L^{\infty}\left(\mathbb{R}^{2}\right)$, and

$$
\sup _{x \in \mathbb{R}^{2}} w(x)|\nabla \phi(x)|+\sup _{x \in \mathbb{R}^{2}} w^{2}(x)\left|\nabla^{2} \phi(x)\right|
$$

is small, where $w(x)=(1+|x|)(1+\ln (1+|x|))$;

(B3) $\left(n_{0}, c_{0}, u_{0}\right)$ satisfies

$$
\begin{gathered}
n_{0}\left(1+|x|+\phi(x)+\left|\ln n_{0}\right|\right) \in L^{1}\left(\mathbb{R}^{2}\right), \\
c_{0} \in L^{1}\left(\mathbb{R}^{2}\right) \cap L^{\infty}\left(\mathbb{R}^{2}\right), \quad \nabla \Psi\left(c_{0}\right) \in L^{2}\left(\mathbb{R}^{2}\right), \quad u_{0} \in L^{2}\left(\mathbb{R}^{2}\right),
\end{gathered}
$$

and $\left\|c_{0}\right\|_{L^{4}}$ is small, where

$$
\Psi(c)=\int_{0}^{c} \sqrt{\frac{\chi(s)}{k(s)}} d s .
$$

If the size of $\phi$ is not small as described in the assumption (B2), we postulate another class of assumptions in which smallness of $c_{0}$ in $L^{\infty}$ is essential:

(C1) $k^{\prime}(c)>0$;

(C2) $\phi=\phi(x) \geq 0, x \in \mathbb{R}^{2}, \nabla \phi \in L^{\infty}\left(\mathbb{R}^{2}\right)$, and

$$
\sup _{x \in \mathbb{R}^{2}} w(x)|\nabla \phi(x)|+\sup _{x \in \mathbb{R}^{2}} w^{2}(x)\left|\nabla^{2} \phi(x)\right|<\infty,
$$

where $w(x)$ is given in (3.7);

(C3) $\left(n_{0}, c_{0}, u_{0}\right)$ satisfies

$$
\begin{gathered}
n_{0}\left(1+|x|+\phi(x)+\left|\ln n_{0}\right|\right) \in L^{1}\left(\mathbb{R}^{2}\right), \\
c_{0} \in L^{1}\left(\mathbb{R}^{2}\right) \cap L^{\infty}\left(\mathbb{R}^{2}\right) \cap H^{1}\left(\mathbb{R}^{2}\right), \quad u_{0} \in L^{2}\left(\mathbb{R}^{2}\right),
\end{gathered}
$$

and $\left\|c_{0}\right\|_{L^{\infty}}$ is small.

Now, the main result of this section is stated in the following

Theorem 3.1. Under the assumptions $(\mathbf{A})(\mathbf{B 1})(\mathbf{B 2})(\mathbf{B 3})$ or $(\mathbf{A})(\mathbf{C 1})(\mathbf{C 2})(\mathbf{C} 3)$, the Cauchy problem (3.1)-(3.2) admits a global weak solution $(\sigma, c, u)$.

The proof of Theorem 3.1 will be carried out as follows. In the next subsection, on the basis of two classes of different assumptions given in Theorem 3.1, for any smooth solutions to the Cauchy problem (3.1)-(3.2), we derive some Lyapunov inequalities in order to obtain the natural a priori bounds uniformly in time described in (i) of Definition 3.1. And then, Theorem 3.1 is proved in Subsection 3.3 due to the usual approximation and compactness argument. Precisely, to establish a complete proof, we firstly regularize initial data $\left(n_{0}^{\epsilon}, c_{0}^{\epsilon}, u_{0}^{\epsilon}\right)$ with $n_{0}^{\epsilon} \in L^{2}\left(\mathbb{R}^{2}\right), c_{0}^{\epsilon} \in H^{1}\left(\mathbb{R}^{2}\right) \cap L^{\infty}\left(\mathbb{R}^{2}\right)$ and $u_{0}^{\epsilon} \in H^{2}\left(\mathbb{R}^{2}\right)$ for $\epsilon>0$. Then, we obtain the global existence of solutions to the corresponding Cauchy problem with the prescribed regularized initial data by designing a mapping over a proper functional space and further applying the Schauder fixed point theorem with the help of the AubinLions compactness method. Finally, based on the obtained a priori estimates, we deal with the existence of weak solutions by passing to the limit $\epsilon \rightarrow 0$ so as to conclude the proof of Theorem 3.1. Here, we remark that Lemma 3.1 and Lemma 3.2 about the uniform a priori estimates also hold for higher space dimensions, and two space dimensions play a key role in the compactness argument when passing to the limit. 


\subsection{Uniform a priori estimates}

The main goal of this subsection is to provide some uniform a priori estimates on any smooth solution to the Cauchy problem (3.1)-(3.2) under the assumptions (A) and (B1)(B2)(B3) or (C1)(C2)(C3). One can achieve this goal if the strength of the potential function $\phi$ is weak or $L^{\infty}$-norm of $c_{0}$ is small.

Lemma 3.1 (case of weak force). Under assumptions (A)(B1)(B2)(B3), any smooth solution $(n, c, u)$ to the Cauchy problem (3.1)-(3.2) satisfies that

$$
\begin{gathered}
n(t, x) \geq 0, c(t, x) \geq 0, t \geq 0, x \in \mathbb{R}^{2} ; \\
\|n(t)\|_{L^{1}} \equiv\left\|n_{0}\right\|_{L^{1}}, t \geq 0 ; \quad \sup _{t \geq 0}\|c(t)\|_{L^{p}} \leq\left\|c_{0}\right\|_{L^{p}}, \quad \text { for any } 1 \leq p \leq \infty,
\end{gathered}
$$

and

$$
\mathcal{E}_{1}(t)+\int_{0}^{t} \mathcal{D}_{1}(s) d s \leq \mathcal{E}_{1}(0),
$$

for any $t \geq 0$, where the temporal free energy functional $\mathcal{E}_{1}(t)$ and its dissipation rate $\mathcal{D}_{1}(t)$ are given by

$$
\begin{aligned}
\mathcal{E}_{1}(t)= & \int_{\mathbb{R}^{2}}\left(n \ln n+\frac{1}{2}|\nabla \Psi(c)|^{2}+\frac{1}{\lambda_{1} \mu \nu} n \phi+\frac{1}{2 \lambda_{1} \mu \nu}|u|^{2}\right) d x, \\
\mathcal{D}_{1}(t)= & \frac{\delta}{2} \int_{\mathbb{R}^{2}} \frac{|\nabla n|^{2}}{n} d x+\frac{\lambda_{0}}{2} \int_{\mathbb{R}^{2}} n|\nabla \Psi|^{2} d x+\frac{\lambda_{1} \mu}{2} \int_{\mathbb{R}^{2}}|\nabla \Psi|^{4} d x+\frac{1}{2 \lambda_{1} \mu} \int_{\mathbb{R}^{2}}|\nabla u|^{2} d x \\
& +\mu \sum_{i j} \int_{\mathbb{R}^{2}}\left|\partial_{i} \partial_{j} \Psi-\frac{d}{d c} \sqrt{\frac{k(c)}{\chi(c)}} \partial_{i} \Psi \partial_{j} \Psi\right|^{2} d x,
\end{aligned}
$$

with constants $\lambda_{0}>0$ and $\lambda_{1}>0$ depending only $\left\|c_{0}\right\|_{\infty}$. Moreover, (n, c, u) satisfies (3.3) for any finite $T>0$.

Proof. Firstly, it is straightforward to obtain (3.9) and (3.10) as before. To prove the Lyapunov inequality (3.11), we claim that the following two identities hold:

$$
\begin{gathered}
\frac{d}{d t} \int_{\mathbb{R}^{2}}\left(n \ln n+\frac{1}{2}|\nabla \Psi(c)|^{2}\right) d x+\delta \int_{\mathbb{R}^{2}} \frac{|\nabla n|^{2}}{n} d x \\
+\int_{\mathbb{R}^{2}} \frac{\chi^{\prime}(c) k(c)+\chi(c) k^{\prime}(c)}{2 \chi(c)} n|\nabla \Psi|^{2} d x+\mu \sum_{i j} \int_{\mathbb{R}^{2}}\left|\partial_{i} \partial_{j} \Psi-\frac{d}{d c} \sqrt{\frac{k(c)}{\chi(c)}} \partial_{i} \Psi \partial_{j} \Psi\right|^{2} d x \\
-\frac{\mu}{2} \int_{\mathbb{R}^{2}} \frac{d^{2}}{d c^{2}}\left(\frac{k(c)}{\chi(c)}\right)|\nabla \Psi|^{4} d x=-\sum_{i j} \int_{\mathbb{R}^{2}} \partial_{i} u_{j} \partial_{i} \Psi \partial_{j} \Psi d x,
\end{gathered}
$$

and

$$
\frac{d}{d t} \int_{\mathbb{R}^{2}}\left(n \phi+\frac{1}{2}|u|^{2}\right) d x+\nu \int_{\mathbb{R}^{2}}|\nabla u|^{2} d x=\delta \int_{\mathbb{R}^{2}} n \Delta \phi d x+\int_{\mathbb{R}^{2}} \sqrt{k(c) \chi(c)} n \nabla \Psi \cdot \nabla \phi d x,
$$

for any $t \geq 0$. In fact, the identity (3.13) directly follows from the equations $(3.1)_{1}$ and $(3.1)_{3}$ and integration by parts. Here, notice that $\phi$ is independent of time $t$, and the definition (3.8) of $\Psi$ was used. To prove (3.12), by the definition (3.8) and the equation $(3.1)_{2}$ of $c, \Psi=\Psi(c)$ satisfies

$$
\partial_{t} \Psi+u \cdot \nabla \Psi=\mu\left[\Delta \Psi+\frac{d}{d c} \sqrt{\frac{k(c)}{\chi(c)}}|\nabla \Psi|^{2}\right]-n \sqrt{k(c) \chi(c)} .
$$


Applying $\nabla$ to the above equation, multiplying it by $\nabla \Psi$ and then integrating, one has

$$
\begin{gathered}
\frac{1}{2} \frac{d}{d t} \int_{\mathbb{R}^{2}}|\nabla \Psi|^{2} d x+\mu \int_{\mathbb{R}^{2}}\left|\nabla^{2} \Psi\right|^{2} d x+\mu \int_{\mathbb{R}^{2}} \frac{d}{d c} \sqrt{\frac{k(c)}{\chi(c)}}|\nabla \Psi|^{2} \Delta \Psi d x \\
=-\sum_{i j} \int_{\mathbb{R}^{2}} \partial_{i} u_{j} \partial_{i} \Psi \partial_{j} \Psi d x+\int_{\mathbb{R}^{2}} \sqrt{k(c) \chi(c)} n \Delta \Psi d x .
\end{gathered}
$$

On the other hand, as before, it follows from $(3.1)_{1}$ that

$$
\begin{aligned}
\frac{d}{d t} \int_{\mathbb{R}^{2}} n \ln n d x+\delta \int_{\mathbb{R}^{2}} \frac{|\nabla n|^{2}}{n} d x & =\int_{\mathbb{R}^{2}} \chi(c) \nabla c \cdot \nabla n d x \\
& =\int_{\mathbb{R}^{2}} \sqrt{k(c) \chi(c)} \nabla \Psi \cdot \nabla n d x
\end{aligned}
$$

which by the further integration by parts, gives

$$
\begin{aligned}
& \frac{d}{d t} \int_{\mathbb{R}^{2}} n \ln n d x+\delta \int_{\mathbb{R}^{2}} \frac{|\nabla n|^{2}}{n} d x \\
& =-\int_{\mathbb{R}^{2}} \sqrt{k(c) \chi(c)} n \Delta \Psi d x-\int_{\mathbb{R}^{2}} \frac{d}{d c} \sqrt{k(c) \chi(c)} \sqrt{\frac{k(c)}{\chi(c)}} n|\nabla \Psi|^{2} d x,
\end{aligned}
$$

Adding (3.15) to (3.14), one has

$$
\begin{aligned}
& \frac{d}{d t} \int_{\mathbb{R}^{2}}\left(n \ln n+\frac{1}{2}|\nabla \Psi|^{2}\right) d x+\delta \int_{\mathbb{R}^{2}} \frac{|\nabla n|^{2}}{n} d x+\mu \int_{\mathbb{R}^{2}}\left|\nabla^{2} \Psi\right|^{2} d x \\
& \quad+\mu \int_{\mathbb{R}^{2}} \frac{d}{d c} \sqrt{\frac{k(c)}{\chi(c)}}|\nabla \Psi|^{2} \Delta \Psi d x+\int_{\mathbb{R}^{2}} \frac{d}{d c} \sqrt{k(c) \chi(c)} \sqrt{\frac{k(c)}{\chi(c)}} n|\nabla \Psi|^{2} d x \\
& =-\sum_{i j} \int_{\mathbb{R}^{2}} \partial_{i} u_{j} \partial_{i} \Psi \partial_{j} \Psi d x,
\end{aligned}
$$

where from the assumptions (A) and (B1), it holds that

$$
\frac{d}{d c} \sqrt{k(c) \chi(c)} \sqrt{\frac{k(c)}{\chi(c)}}=\frac{\chi^{\prime}(c) k(c)+\chi(c) k^{\prime}(c)}{2 \chi(c)}>0 .
$$

Noticing the identity

$$
\nabla \cdot\left(|\nabla \Psi|^{2} \nabla \Psi\right)=|\nabla \Psi|^{2} \Delta \Psi+\nabla\left(|\nabla \Psi|^{2}\right) \cdot \nabla \Psi,
$$

it follows from integration by parts that

$$
\begin{aligned}
\mu \int_{\mathbb{R}^{2}} \frac{d}{d c} \sqrt{\frac{k(c)}{\chi(c)}}|\nabla \Psi|^{2} \Delta \Psi d x= & -\mu \int_{\mathbb{R}^{2}} \frac{d^{2}}{d c^{2}} \sqrt{\frac{k(c)}{\chi(c)}} \sqrt{\frac{k(c)}{\chi(c)}}|\nabla \Psi|^{4} d x \\
& -2 \mu \sum_{i j} \int_{\mathbb{R}^{2}} \frac{d}{d c} \sqrt{\frac{k(c)}{\chi(c)}} \partial_{i} \Psi \partial_{j} \Psi \partial_{i} \partial_{j} \Psi d x
\end{aligned}
$$


Therefore, it holds that

$$
\begin{aligned}
& \mu \int_{\mathbb{R}^{2}}\left|\nabla^{2} \Psi\right|^{2} d x+\mu \int_{\mathbb{R}^{2}} \frac{d}{d c} \sqrt{\frac{k(c)}{\chi(c)}}|\nabla \Psi|^{2} \Delta \Psi d x \\
& =\mu \sum_{i j} \int_{\mathbb{R}^{2}}\left(\left|\partial_{i} \partial_{j} \Psi\right|^{2}-2 \frac{d}{d c} \sqrt{\frac{k(c)}{\chi(c)}} \partial_{i} \Psi \partial_{j} \Psi \partial_{i} \partial_{j} \Psi-\frac{d^{2}}{d c^{2}} \sqrt{\frac{k(c)}{\chi(c)}} \sqrt{\frac{k(c)}{\chi(c)}}\left|\partial_{i} \Psi\right|^{2}\left|\partial_{j} \Psi\right|^{2}\right) d x \\
& =\mu \sum_{i j} \int_{\mathbb{R}^{2}}\left|\partial_{i} \partial_{j} \Psi-\frac{d}{d c} \sqrt{\frac{k(c)}{\chi(c)}} \partial_{i} \Psi \partial_{j} \Psi\right|^{2} d x-\frac{1}{2} \mu \int_{\mathbb{R}^{2}} \frac{d^{2}}{d c^{2}} \frac{k(c)}{\chi(c)}|\nabla \Psi|^{4} d x
\end{aligned}
$$

where we used the identity

$$
\left(\frac{d}{d c} \sqrt{\frac{k(c)}{\chi(c)}}\right)^{2}+\frac{d^{2}}{d c^{2}} \sqrt{\frac{k(c)}{\chi(c)}} \sqrt{\frac{k(c)}{\chi(c)}}=\frac{1}{2} \frac{d^{2}}{d c^{2}}\left(\frac{k(c)}{\chi(c)}\right) .
$$

Thus, putting (3.17) into (3.16) yields the desired identity (3.12).

Now, based on the identities (3.12) and (3.13), one can prove the Lyapunov inequality (3.11). In fact, let us denote $c_{M}=\left\|c_{0}\right\|_{L^{\infty}}$ and define

$$
\begin{aligned}
\lambda_{0} & =\min _{0 \leq c \leq c_{M}} \frac{\chi^{\prime}(c) k(c)+\chi(c) k^{\prime}(c)}{2 \chi(c)}, \\
\lambda_{1} & =\min _{0 \leq c \leq c_{M}}-\frac{1}{2} \frac{d^{2}}{d c^{2}}\left(\frac{k(c)}{\chi(c)}\right),
\end{aligned}
$$

where by the assumptions (A) and (B1), $\lambda_{0}>0$ and $\lambda_{1}>0$ hold true. Thus from the identity (3.12) and the Cauchy-Schwarz inequality, one has

$$
\begin{aligned}
& \frac{d}{d t} \int_{\mathbb{R}^{2}}\left(n \ln n+\frac{1}{2}|\nabla \Psi(c)|^{2}\right) d x+\delta \int_{\mathbb{R}^{2}} \frac{|\nabla n|^{2}}{n} d x+\lambda_{0} \int_{\mathbb{R}^{2}} n|\nabla \Psi|^{2} d x \\
& \quad+\mu \sum_{i j} \int_{\mathbb{R}^{2}}\left|\partial_{i} \partial_{j} \Psi-\frac{d}{d c} \sqrt{\frac{k(c)}{\chi(c)}} \partial_{i} \Psi \partial_{j} \Psi\right|^{2} d x+\frac{\lambda_{1} \mu}{2} \int_{\mathbb{R}^{2}}|\nabla \Psi|^{4} d x \\
& \leq \frac{1}{2 \lambda_{1} \mu} \int_{\mathbb{R}^{2}}|\nabla u|^{2} d x .
\end{aligned}
$$

Take $\epsilon_{\phi}>0$ and suppose

$$
\sup _{x} w(x)|\nabla \phi(x)|+\sup _{x} w^{2}(x)|\Delta \phi(x)| \leq \epsilon_{\phi} .
$$

Then similarly, from (3.13), one has

$$
\begin{aligned}
& \frac{d}{d t} \int_{\mathbb{R}^{2}}\left(n \phi+\frac{1}{2}|u|^{2}\right) d x+\nu \int_{\mathbb{R}^{2}}|\nabla u|^{2} d x \\
& \leq \delta \epsilon_{\phi} \int_{\mathbb{R}^{2}}\left|\frac{\sqrt{n}}{w(x)}\right|^{2} d x+\epsilon_{\phi}\left(\sup _{0 \leq c \leq c_{M}} k(c) \chi(c)\right)^{1 / 2} \int_{\mathbb{R}^{2}} \frac{\sqrt{n}}{w(x)} \cdot \sqrt{n}|\nabla \Psi| d x \\
& \leq\left(\delta \epsilon_{\phi}+\frac{\sup _{0 \leq c \leq c_{M}} k(c) \chi(c)}{2 \lambda_{0} \lambda_{1} \mu \nu} \epsilon_{\phi}^{2}\right) \int_{\mathbb{R}^{2}} \frac{|\nabla n|^{2}}{n} d x+\frac{\lambda_{0} \lambda_{1} \mu \nu}{2} \int_{\mathbb{R}^{2}} n|\nabla \Psi|^{2} d x,
\end{aligned}
$$

where one used the Hardy inequality over $\mathbb{R}^{2}$

$$
\int_{\mathbb{R}^{2}}\left|\frac{\sqrt{n}}{w(x)}\right|^{2} d x \leq C \int_{\mathbb{R}^{2}}|\nabla \sqrt{n}|^{2} d x \leq C \int_{\mathbb{R}^{2}} \frac{|\nabla n|^{2}}{n} d x
$$


for $w(x)=(1+|x|)(1+\ln (1+|x|))$. One can choose $\epsilon_{\phi}>0$ small enough such that

$$
\frac{1}{\lambda_{1} \mu \nu}\left(\delta \epsilon_{\phi}+\frac{\sup _{0 \leq c \leq c_{M}} k(c) \chi(c)}{2 \lambda_{0} \lambda_{1} \mu \nu} \epsilon_{\phi}^{2}\right) \leq \frac{\delta}{2} .
$$

Therefore, (3.11) follows by multiplying (3.19) by $1 /\left(\lambda_{1} \mu \nu\right)$ and then adding it to (3.18). Thus (3.11) is proved.

Finally, we prove that $(n, c, u)$ satisfies (3.3) for any finite $T>0$. The key point is to obtain the bound of the first-order spatial moment of $n(t, x)$. For this purpose, multiplying $(3.1)_{1}$ by the smooth function $\langle x\rangle=\left(1+|x|^{2}\right)^{1 / 2}$ and taking integration, one has

$$
\frac{d}{d t} \int_{\mathbb{R}^{2}}\langle x\rangle n d x=\int_{\mathbb{R}^{2}} n u \cdot \nabla\langle x\rangle d x+\delta \int_{\mathbb{R}^{2}} n \Delta\langle x\rangle d x+\int_{\mathbb{R}^{2}} \sqrt{k(c) \chi(c)} n \nabla \Psi \cdot \nabla\langle x\rangle d x .
$$

Next, we estimate each term on the r.h.s. of the above identity. Notice that $\|\nabla\langle x\rangle\|_{L^{\infty}}$ and $\|\Delta\langle x\rangle\|_{L^{\infty}}$ are finite constants. For the first term, it follows from the Cauchy-Schwarz inequality, Sobolev inequality and the mass conservation for $n$ that

$$
\begin{aligned}
\int_{\mathbb{R}^{2}} n u \cdot \nabla \varphi d x & \leq\|\nabla\langle x\rangle\|_{L^{\infty}}\|n\|_{L^{2}}\|u\|_{L^{2}} \leq C\|\sqrt{n}\|_{L^{4}}^{2}\|u\|_{L^{2}} \leq C\|\sqrt{n}\|_{L^{2}}\|\nabla \sqrt{n}\|_{L^{2}}\|u\|_{L^{2}} \\
& \leq \epsilon\|\nabla \sqrt{n}\|^{2}+\frac{C\left\|n_{0}\right\|_{L^{1}}}{\epsilon}\|u\|^{2},
\end{aligned}
$$

where $\epsilon>0$ is a small constant to be chosen later. For the second term, it is straightforward to use the mass conservation for $n$ to obtain

$$
\delta \int_{\mathbb{R}^{2}} n \Delta\langle x\rangle d x \leq \delta\|\Delta \varphi\|_{L^{\infty}}\|n\|_{L^{1}} \leq C \delta\left\|n_{0}\right\|_{L^{1}} .
$$

For the third term, it follows from the Cauchy-Schwarz inequality that

$$
\int_{\mathbb{R}^{2}} \sqrt{k(c) \chi(c)} n \nabla \Psi \cdot \nabla\langle x\rangle d x \leq \epsilon\|\sqrt{n} \nabla \Psi\|^{2}+\frac{C}{\epsilon}\left(\sup _{0 \leq c \leq c_{M}} \chi(c)\right)\|\sqrt{k(c) n}\|^{2} .
$$

Collecting the above estimates, it therefore holds that

$$
\begin{aligned}
\frac{d}{d t} \int_{\mathbb{R}^{2}}\langle x\rangle n d x \leq & C \delta\left\|n_{0}\right\|_{L^{1}}+\frac{C}{\epsilon}\left(\sup _{0 \leq c \leq c_{M}} \chi(c)\right)\|k(c) n\|_{L^{1}}+\frac{C\left\|n_{0}\right\|_{L^{1}}}{\epsilon}\|u\|_{L^{2}}^{2} \\
& +\epsilon\left(\|\nabla \sqrt{n}\|_{L^{2}}^{2}+\|\sqrt{n} \nabla \Psi\|^{2}\right) .
\end{aligned}
$$

On the other hand, recall from the proof of (3.11) that for the free energy functional $\mathcal{E}_{1}(t)$ and the corresponding non-negative dissipation rate $\mathcal{D}_{1}(t)$, one has

$$
\frac{d}{d t} \mathcal{E}_{1}(t)+\mathcal{D}_{1}(t) \leq 0
$$

for any $t \geq 0$. Now, let us define a modified temporal functional $\mathcal{E}_{1}^{+}(t)$ from $\mathcal{E}_{1}(t)$ by

$$
\mathcal{E}_{1}^{+}(t)=\mathcal{E}_{1}(t)+\Lambda \int_{\mathbb{R}^{2}}\langle x\rangle n d x,
$$

where $\Lambda>0$ is a large constant to be chosen later. We claim that as long as $\Lambda>0$ is large enough, it holds that

$$
\mathcal{E}_{1}^{+}(t) \sim \int_{\mathbb{R}^{2}}\left[n(|\ln n|+\langle x\rangle+\phi(x))+|\nabla \Psi(c)|^{2}+|u|^{2}\right] d x,
$$


where $A \sim B$ means that $C_{1} B \leq A \leq C_{2} B$ holds for two generic constants $C_{1}>0$ and $C_{2}>0$. In fact, this follows from the identity

$$
\int_{\mathbb{R}^{2}} n \ln n d x=\int_{\mathbb{R}^{2}} n|\ln n| d x-2 \int_{\mathbb{R}^{2}} n \ln \frac{1}{n} \chi_{n \leq 1} d x,
$$

and the estimate

$$
\begin{aligned}
0 \leq \int_{\mathbb{R}^{2}} n \ln \frac{1}{n} \chi_{n \leq 1} d x & =\int_{\mathbb{R}^{2}} n \ln \frac{1}{n} \chi_{e^{-\langle x\rangle} \leq n} d x+\int_{\mathbb{R}^{2}} n \ln \frac{1}{n} \chi_{n \leq e^{-\langle x\rangle}} d x \\
& \leq \int_{\mathbb{R}^{2}}\langle x\rangle n d x+C \int_{\mathbb{R}^{2}} n^{1 / 2} \chi_{n \leq e^{-\langle x\rangle}} d x \\
& \leq \int_{\mathbb{R}^{2}}\langle x\rangle n d x+C \\
& \leq\left(1+\frac{C}{\left\|n_{0}\right\|_{L^{1}}}\right) \int_{\mathbb{R}^{2}}\langle x\rangle n d x,
\end{aligned}
$$

where $\left\|n_{0}\right\|_{L^{1}}>0$ has been assumed without loss of generality and the mass conservation law for $n$ was also used. Then, one can fix the constant $\Lambda>0$ in (3.22) large enough, depending only on the total mass $\left\|n_{0}\right\|_{L^{1}}$ of cells, such that (3.23) holds. Recall the definition of $\mathcal{D}_{1}(t)$ in Lemma 3.1. Then, by letting $\epsilon>0$ small enough, the linear combination of (3.20) and (3.21) yields

$$
\leq \begin{gathered}
\frac{d}{d t} \mathcal{E}_{1}^{+}(t)+\frac{1}{2} \mathcal{D}_{1}(t) \\
\leq C \Lambda \delta\left\|n_{0}\right\|_{L^{1}}+\frac{C \Lambda}{\epsilon}\left(\sup _{0 \leq c \leq c_{M}} \chi(c)\right)\|\sqrt{k(c) n}\|^{2}+\frac{C \Lambda\left\|n_{0}\right\|_{L^{1}}}{\epsilon} \mathcal{E}_{1}^{+}(t),
\end{gathered}
$$

for any $t \geq 0$, where (3.23) was used. Recall that from the equation $(3.1)_{2}$, it follows that

$$
\|c(t)\|_{L^{1}}+\int_{0}^{t}\|k(c) n\|_{L^{1}} d s \leq\left\|c_{0}\right\|_{L^{1}},
$$

for any $t \geq 0$. Then, applying the Gronwall inequality to (3.24), one has

$$
\begin{aligned}
\mathcal{E}_{1}^{+}(t) \leq & \mathcal{E}_{1}^{+}(0) e^{\frac{C \Lambda T}{\epsilon}\left\|n_{0}\right\|_{L^{1}}} \\
& +\int_{0}^{t}\left[C \Lambda \delta\left\|n_{0}\right\|_{L^{1}}+\frac{C \Lambda}{\epsilon}\left(\sup _{0 \leq c \leq c_{M}} \chi(c)\right)\|k(c) n\|_{L^{1}}\right] d s e^{\frac{C \Lambda T}{\epsilon}\left\|n_{0}\right\|_{L^{1}}} \\
\leq & {\left[\mathcal{E}_{1}^{+}(0)+C \Lambda \delta\left\|n_{0}\right\|_{L^{1}} T+\frac{C \Lambda}{\epsilon}\left\|c_{0}\right\|_{L^{1}} \sup _{0 \leq c \leq c_{M}} \chi(c)\right] e^{\frac{C \Lambda T}{\epsilon}\left\|n_{0}\right\|_{L^{1}}}, }
\end{aligned}
$$

for any $0 \leq t \leq T$. Therefore, the time integration of (3.24) gives

$$
\mathcal{E}_{1}^{+}(t)+\int_{0}^{t} \mathcal{D}_{1}(s) d s \leq C\left(n_{0}, c_{0}, u_{0}, T\right)
$$

for any $0 \leq t \leq T$, where $C\left(n_{0}, c_{0}, u_{0}, T\right)$ is a finite constant depending only on $T$ and bounds of $\left(n_{0}, c_{0}, u_{0}\right)$ appearing in the assumption (B3). Finally, similarly as before, from $(3.1)_{2}$, one has

$$
\|c(t)\|^{2}+2 \mu \int_{0}^{t}\|\nabla c(s)\| d s \leq\left\|c_{0}\right\|^{2},
$$

for any $t \geq 0$. Notice

$$
|\nabla \Psi(c)|^{2}=\frac{\chi(c)}{k(c)}|\nabla c|^{2} \geq \lambda_{3}|\nabla c|^{2}
$$


where from the assumptions (A) and (B1), $\lambda_{3}$ is a positive constant defined by

$$
\lambda_{3}=\frac{\min _{0 \leq c \leq c_{M}} \chi(c)}{\max _{0 \leq c \leq c_{M}} k(c)}>0 .
$$

Hence, (3.3) follows from (3.25), (3.26) and (3.27). This completes the proof of Lemma 3.1.

Lemma 3.2 (case of small $\left.c_{0}\right)$. Under assumptions $(\mathbf{A})(\mathbf{C 1})(\mathbf{C 2})(\mathbf{C} 3)$, any smooth solution $(n, c, u)$ to the Cauchy problem (3.1)-(3.2) satisfies that

$$
\begin{gathered}
n(t, x) \geq 0, c(t, x) \geq 0, t \geq 0, x \in \mathbb{R}^{2} ; \\
\|n(t)\|_{L^{1}} \equiv\left\|n_{0}\right\|_{L^{1}}, t \geq 0 ; \quad \sup _{t \geq 0}\|c(t)\|_{L^{p}} \leq\left\|c_{0}\right\|_{L^{p}}, \quad \text { for any } 1 \leq p \leq \infty,
\end{gathered}
$$

and

$$
\mathcal{E}_{2}(t)+\lambda \int_{0}^{t} \mathcal{D}_{2}(s) d s \leq C\left(\left\|n_{0} \ln n_{0}\right\|_{L^{1}}+\left\|c_{0}\right\|_{H^{1}}^{2}+\left\|u_{0}\right\|_{L^{2}}^{2}\right),
$$

for any $t \geq 0$, where the free energy functional $\mathcal{E}_{2}(t)$ and its dissipation rate $\mathcal{D}_{2}(t)$ are given by

$$
\begin{aligned}
\mathcal{E}_{2}(t) & =\int_{\mathbb{R}^{2}} n(\ln n+\lambda \phi) d x+\lambda\left(\|c\|_{H^{1}}^{2}+\|u\|^{2}\right) \\
\mathcal{D}_{2}(t) & =\|\nabla \sqrt{n}\|^{2}+\|\nabla c\|_{H^{1}}^{2}+\|\sqrt{n} c\|^{2}+\|\sqrt{n} \nabla c\|^{2}+\|\nabla u\|^{2},
\end{aligned}
$$

with $\lambda>0$ a small constant. Moreover, $(n, c, u)$ also satisfies (3.3) for any finite $T>0$.

Proof. It suffices to prove the Lyapunov inequality (3.28) since the rest estimates can be obtained similarly as in the proof of Lemma 3.1. To the end, we still denote $c_{M}=\left\|c_{0}\right\|_{L^{\infty}}$. Firstly, similarly as before, from $(3.1)_{1}$, it holds that

$$
\begin{aligned}
\frac{d}{d t} \int_{\mathbb{R}^{2}} n \ln n d x & +\delta \int_{\mathbb{R}^{2}} \frac{|\nabla n|^{2}}{n} d x=\int_{\mathbb{R}^{2}} \chi(c) \nabla c \cdot \nabla n d x \\
& \leq \frac{\delta}{2} \int_{\mathbb{R}^{2}} \frac{|\nabla n|^{2}}{n} d x+\frac{1}{2 \delta} \max _{0 \leq c \leq c_{M}}|\chi(c)|^{2} \int_{\mathbb{R}^{2}} n|\nabla c|^{2} d x,
\end{aligned}
$$

which implies that

$$
\frac{d}{d t} \int_{\mathbb{R}^{2}} n \ln n d x+\frac{\delta}{2} \int_{\mathbb{R}^{2}} \frac{|\nabla n|^{2}}{n} d x \leq \frac{1}{2 \delta} \max _{0 \leq c \leq c_{M}}|\chi(c)|^{2} \int_{\mathbb{R}^{2}} n|\nabla c|^{2} d x .
$$

From $(3.1)_{2}$, it follows that

$$
\frac{1}{2} \frac{d}{d t} \int_{\mathbb{R}^{2}}|c|^{2} d x+\mu \int_{\mathbb{R}^{2}}|\nabla c|^{2} d x+\int_{\mathbb{R}^{2}} k(c) c n d x=0,
$$

and

$$
\begin{gathered}
\frac{1}{2} \frac{d}{d t} \int_{\mathbb{R}^{2}}|\nabla c|^{2} d x+\mu \int_{\mathbb{R}^{2}}\left|\nabla^{2} c\right|^{2} d x+\min _{0 \leq c \leq c_{M}} k^{\prime}(c) \int_{\mathbb{R}^{2}} n|\nabla c|^{2} d x \\
\leq \eta \int_{\mathbb{R}^{2}} n|\nabla c|^{2} d x+\frac{1}{4 \eta} \int_{\mathbb{R}^{2}}|k(c)|^{2} \frac{|\nabla n|^{2}}{n} d x+\frac{\mu}{2} \int_{\mathbb{R}^{2}}\left|\nabla^{2} c\right|^{2} d x+\frac{1}{2 \mu} \int_{\mathbb{R}^{2}}|\nabla u|^{2} c^{2} d x,
\end{gathered}
$$

for any $\eta>0$, where it is noticed that

$$
\min _{0 \leq c \leq c_{M}} k^{\prime}(c)>0
$$


due to the assumption (C1). By taking $\eta=\frac{1}{2} \min _{0 \leq c \leq c_{M}} k^{\prime}(c)$, one has

$$
\begin{aligned}
& \frac{d}{d t} \int_{\mathbb{R}^{2}}|\nabla c|^{2} d x+\mu \int_{\mathbb{R}^{2}}\left|\nabla^{2} c\right|^{2} d x+\min _{0 \leq c \leq c_{M}} k^{\prime}(c) \int_{\mathbb{R}^{2}} n|\nabla c|^{2} d x \\
& \leq \frac{\max _{0 \leq c \leq c_{M}}|k(c)|^{2}}{\min _{0 \leq c \leq c_{M}} k^{\prime}(c)} \int_{\mathbb{R}^{2}} \frac{|\nabla n|^{2}}{n} d x+\frac{c_{M}^{2}}{\mu} \int_{\mathbb{R}^{2}}|\nabla u|^{2} d x .
\end{aligned}
$$

Let $\lambda_{4}>0$ be such that

$$
\lambda_{4} \min _{0 \leq c \leq c_{M}} k^{\prime}(c)=\frac{1}{2 \delta} \max _{0 \leq c \leq c_{M}}|\chi(c)|^{2}+1,
$$

and then let $c_{M}$ be small enough due to the assumption (C3), such that

$$
\lambda_{4} \frac{\max _{0 \leq c \leq c_{M}}|k(c)|^{2}}{\min _{0 \leq c \leq c_{M}} k^{\prime}(c)} \leq \frac{\delta}{4}
$$

where $k(0)=0$ from the assumption (A) was used. Thus, multiplying (3.31) by $\lambda_{4}$ and adding it to (3.29) gives

$$
\begin{aligned}
\frac{d}{d t} \int_{\mathbb{R}^{2}}\left(n \ln n+\lambda_{4}|\nabla c|^{2}\right) d x & +\lambda_{4} \mu \int_{\mathbb{R}^{2}}\left|\nabla^{2} c\right|^{2} d x+\int_{\mathbb{R}^{2}} n|\nabla c|^{2} d x \\
& +\frac{\delta}{4} \int_{\mathbb{R}^{2}} \frac{|\nabla n|^{2}}{n} d x \leq \frac{\lambda_{4} c_{M}^{2}}{\mu} \int_{\mathbb{R}^{2}}|\nabla u|^{2} d x,
\end{aligned}
$$

which further combining with (3.30) yields

$$
\begin{aligned}
& \frac{d}{d t} \int_{\mathbb{R}^{2}}\left(n \ln n+\lambda_{4}|\nabla c|^{2}+|c|^{2}\right) d x+\mu \min \left\{\lambda_{4}, 2\right\} \int_{\mathbb{R}^{2}}\left(|\nabla c|^{2}+\left|\nabla^{2} c\right|^{2}\right) d x \\
& \quad+\min \left\{1,2 \min _{0 \leq c \leq c_{M}} k^{\prime}(c)\right\} \int_{\mathbb{R}^{2}} n\left(|c|^{2}+|\nabla c|^{2}\right) d x+\frac{\delta}{4} \int_{\mathbb{R}^{2}} \frac{|\nabla n|^{2}}{n} d x \\
& \leq \frac{\lambda_{4} c_{M}^{2}}{\mu} \int_{\mathbb{R}^{2}}|\nabla u|^{2} d x .
\end{aligned}
$$

Similarly as before, from $(3.1)_{3}$, it holds that

$$
\begin{aligned}
& \frac{d}{d t} \int_{\mathbb{R}^{2}}\left(n \phi+\frac{1}{2}|u|^{2}\right) d x+\nu \int_{\mathbb{R}^{2}}|\nabla u|^{2} d x=\delta \int_{\mathbb{R}^{2}} n \Delta \phi d x+\int_{\mathbb{R}^{2}} \chi(c) n \nabla c \cdot \nabla \phi d x \\
& \leq \delta \sup _{x} w(x)^{2}|\Delta \phi(x)| \int_{\mathbb{R}^{2}} \frac{|\nabla n|^{2}}{n} d x \\
& \quad+\frac{1}{2} \sup _{x} w(x)|\nabla \phi(x)| \sup _{0 \leq c \leq c_{M}}|\chi(c)|\left(\int_{\mathbb{R}^{2}} \frac{|\nabla n|^{2}}{n} d x+\int_{\mathbb{R}^{2}} n|\nabla c|^{2} d x\right) .
\end{aligned}
$$

Therefore, multiplying (3.33) by a small constant $\lambda_{5}>0$, adding it to (3.32) and then taking $c_{M}$ small enough, one has

$$
\begin{aligned}
& \frac{d}{d t} \int_{\mathbb{R}^{2}}\left[n \ln n+\lambda_{4}|\nabla c|^{2}+|c|^{2}+\lambda_{5}\left(n \phi+\frac{1}{2}|u|^{2}\right)\right] d x \\
& \quad+\mu \min \left\{\lambda_{4}, 2\right\} \int_{\mathbb{R}^{2}}\left(|\nabla c|^{2}+\left|\nabla^{2} c\right|^{2}\right) d x+\min \left\{\frac{1}{2}, \min _{0 \leq c \leq c_{M}} k^{\prime}(c)\right\} \int_{\mathbb{R}^{2}} n\left(|c|^{2}+|\nabla c|^{2}\right) d x \\
& \quad+\frac{\delta}{8} \int_{\mathbb{R}^{2}} \frac{|\nabla n|^{2}}{n} d x+\frac{\lambda_{5} \nu}{2} \int_{\mathbb{R}^{2}}|\nabla u|^{2} d x \leq 0,
\end{aligned}
$$

for any $t \geq 0$. Hence, (3.28) follows by further taking the time integral of the above inequality. This completes the proof of Lemma 3.2. 


\subsection{Proof of global existence}

In this subsection, we devote ourselves to the proof of Theorem 3.1 by three steps.

Step 1 Construction of the regularized solutions. Given initial data $\left(n_{0}, c_{0}, u_{0}\right)$ satisfying the assumption of Theorem 3.1, let $\left(n_{0}^{\epsilon}, c_{0}^{\epsilon}, u_{0}^{\epsilon}\right)$ for $\epsilon>0$ be a smooth approximation of $\left(n_{0}, c_{0}, u_{0}\right)$, where

$$
n_{0}^{\epsilon} \in L^{2}\left(\mathbb{R}^{2}\right), c_{0}^{\epsilon} \in H^{1}\left(\mathbb{R}^{2}\right) \cap L^{\infty}\left(\mathbb{R}^{2}\right), u_{0}^{\epsilon} \in H^{2}\left(\mathbb{R}^{2}\right),
$$

and

$$
\begin{gathered}
n_{0}^{\epsilon}(x) \geq 0, c_{0}^{\epsilon}(x) \geq 0, \nabla \cdot u_{0}^{\epsilon}(x)=0, x \in \mathbb{R}^{2}, \\
n_{0}^{\epsilon}\left(1+|x|^{2}+\left|\ln n_{0}^{\epsilon}\right|\right) \in L^{1}\left(\mathbb{R}^{2}\right),
\end{gathered}
$$

hold, and it is further supposed that one has the convergence

$$
\begin{gathered}
n_{0}^{\epsilon} \rightarrow n_{0} \text { in } L^{1}\left(\mathbb{R}^{2}\right), c_{0}^{\epsilon} \rightarrow c_{0} \text { in } L^{p}\left(\mathbb{R}^{2}\right)(1 \leq p<\infty), c_{0}^{\epsilon} \rightarrow c_{0} \text { in } \mathrm{w}^{*}-L^{\infty}\left(\mathbb{R}^{2}\right), \\
\nabla c_{0}^{\epsilon} \rightarrow \nabla c_{0} \text { in } L^{2}\left(\mathbb{R}^{2}\right), u_{0}^{\epsilon} \rightarrow u_{0} \text { in } L^{2}\left(\mathbb{R}^{2}\right),
\end{gathered}
$$

as $\epsilon$ tends to zero. Consider the Cauchy problem

$$
\left\{\begin{array}{l}
\partial_{t} n^{\epsilon}+u^{\epsilon} \cdot \nabla n^{\epsilon}=\delta \Delta n^{\epsilon}-\nabla \cdot\left(\chi\left(c^{\epsilon}\right) n^{\epsilon} \nabla c^{\epsilon}\right), \\
\partial_{t} c^{\epsilon}+u^{\epsilon} \cdot \nabla c^{\epsilon}=\mu \Delta c^{\epsilon}-k\left(c^{\epsilon}\right) n^{\epsilon}, \\
\partial_{t} u^{\epsilon}+\nabla P^{\epsilon}=\nu \Delta u^{\epsilon}-n^{\epsilon} \nabla \phi, \\
\nabla \cdot u^{\epsilon}=0, \quad t>0, x \in \mathbb{R}^{2},
\end{array}\right.
$$

with the prescribed-above initial data

$$
\left.\left(n^{\epsilon}, c^{\epsilon}, u^{\epsilon}\right)\right|_{t=0}=\left(n_{0}^{\epsilon}(x), c_{0}^{\epsilon}(x), u_{0}^{\epsilon}(x)\right), \quad x \in \mathbb{R}^{2} .
$$

Now, one can state the global existence result for the regularized solutions with large initial data.

Proposition 3.1. Suppose that either assumptions (A)(B1)(B2)(B3) or (A)(C1)(C2)(C3) hold. Fix $\epsilon>0$. Let (3.34), (3.35) and (3.36) hold. Then, for any finite $T>0$, the Cauchy problem (3.39)-(3.40) admits one solution $\left(n^{\epsilon}, c^{\epsilon}, u^{\epsilon}\right)$ with $n^{\epsilon}(t, x) \geq 0, c^{\epsilon}(t, x) \geq 0,0 \leq t \leq T, x \in \mathbb{R}^{2}$, and

$$
\begin{aligned}
n^{\epsilon} & \in C\left([0, T] ; L^{2}\left(\mathbb{R}^{2}\right)\right) \cap L^{2}\left(0, T ; H^{1}\left(\mathbb{R}^{2}\right)\right), \\
c^{\epsilon} & \in C\left([0, T] ; H^{1}\left(\mathbb{R}^{2}\right)\right) \cap L^{2}\left(0, T ; H^{2}\left(\mathbb{R}^{2}\right)\right) \cap L^{\infty}\left((0, T) \times \mathbb{R}^{2}\right), \\
u^{\epsilon} & \in C\left([0, T] ; H^{2}\left(\mathbb{R}^{2}\right)\right) \cap L^{2}\left(0, T ; H^{3}\left(\mathbb{R}^{2}\right)\right) .
\end{aligned}
$$

Proof. Fix $T>0$. For this time we shall skip the parameter $\epsilon>0$ in (3.39)-(3.40) for simplicity. Denote $H=L^{2}\left(\mathbb{R}^{2}\right)$ and

$$
\begin{aligned}
V & =\left\{\rho \in H^{1}\left(\mathbb{R}^{2}\right) ;\left\||x|^{2} \rho\right\|_{L^{1}}<\infty\right\}, \\
Y(0, T) & =\left\{\rho \in L^{2}(0, T ; V) ; \partial_{t} \rho \in L^{2}\left(0, T ; V^{\prime}\right), \rho(t, x) \geq 0,0 \leq t \leq T, x \in \mathbb{R}^{2}\right\},
\end{aligned}
$$

where $V^{\prime}$ is the dual of $V$. Define a mapping $\mathcal{T}: Y(0, T) \subset L^{2}(0, T ; H) \rightarrow L^{2}(0, T ; H)$ as follows. Given $0 \leq \widetilde{n} \in Y(0, T), n=: \mathcal{T}[\widetilde{n}]$ is obtained by iteratively solving three Cauchy problems:

(i) Solve $u=: \mathcal{T}_{1}[\widetilde{n}]$ to be the solution to the Cauchy problem

$$
\left\{\begin{array}{l}
\partial_{t} u+\nabla P=\nu \Delta u-\widetilde{n} \nabla \phi, \\
\nabla \cdot u=0 \\
\left.u\right|_{t=0}=u_{0} .
\end{array}\right.
$$


Actually, $u$ can be given by

$$
u(t)=G(t) * u_{0}+\int_{0}^{t} G(t-s) * \mathbf{P}(-\widetilde{n} \nabla \phi) d s,
$$

where $\mathbf{P}$ is the divergence-free projection operator in $\mathbb{R}^{2}, G(t, x)=(4 \pi t)^{-1} e^{-\frac{|x|^{2}}{4 t}}$ is the Green function associated to the heat equation in $\mathbb{R}^{2}$ and $*$ denotes the space convolution. By the standard energy estimates on the Stokes system and the Hardy inequality over $\mathbb{R}^{2}$, it follows that

$$
\|u(t)\|_{H^{2}}^{2}+\lambda \int_{0}^{t}\left(\|\nabla u\|_{H^{2}}^{2}+\left\|\partial_{t} u\right\|^{2}\right) d s \leq\left\|u_{0}\right\|_{H^{2}}^{2}+C\left(M_{\phi}\right) \int_{0}^{t}\|\widetilde{n}\|_{H^{1}}^{2} d s,
$$

for any $0 \leq t \leq T$, where $M_{\phi}$ denotes the bound of $\phi$ :

$$
M_{\phi}=\sup _{x \in \mathbb{R}^{2}} w(x)|\nabla \phi(x)|+\sup _{x \in \mathbb{R}^{2}} w^{2}(x)\left|\nabla^{2} \phi(x)\right|,
$$

and $C(\cdot)$ is a nondecreasing continuous function with $C(0)=0$. Therefore, one has

$$
u^{\epsilon} \in C\left([0, T] ; H^{2}\left(\mathbb{R}^{2}\right)\right) \cap L^{2}\left(0, T ; H^{3}\left(\mathbb{R}^{2}\right)\right),
$$

where the continuity follows from the similar proof as that of Lemma 2.2.

(ii) Solve $c=: \mathcal{T}_{2}[\widetilde{n}]$ to be the solution to the Cauchy problem

$$
\left\{\begin{array}{l}
\partial_{t} c+u \cdot \nabla c=\mu \Delta c-k(c) \tilde{n} \\
\left.c\right|_{t=0}=c_{0}
\end{array}\right.
$$

where $u=\mathcal{T}_{1}[\widetilde{n}]$ is given in (i). For that, define the iterative scheme by

$$
\partial_{t} c^{j+1}+u \cdot \nabla c^{j}=\mu \Delta c^{j+1}-k\left(c^{j}\right) \widetilde{n},
$$

for $j \geq 0$, where $c^{0} \equiv 0$ has been set. Notice that $c^{j+1}$ can be written as

$$
c^{j+1}(t)=G(t) * c_{0}+\int_{0}^{t} \nabla G(t-s) *\left(u(s) c^{j}(s)\right) d s-\int_{0}^{t} G(t-s)\left(k\left(c^{j}(s)\right) \widetilde{n}(s)\right) d s,
$$

for any $0 \leq t \leq T$.

The convergence of the sequence $\left(c^{j}\right)_{j \geq 0}$ is verified as follows. Without loss of generality we may suppose $c_{0} \in H^{2}\left(\mathbb{R}^{2}\right)$. Take $T_{0}>0$ small enough. Similar to the proof in Subsection 2.3, we use induction to prove that there are constants $\lambda>0, M>0$ such that

$$
\left\|c^{j}(t)\right\|_{H^{2}}+\lambda \int_{0}^{t}\left\|\nabla c^{j}\right\|_{H^{3}}^{2} d s \leq M
$$

for any $j \geq 0$ and $0 \leq t \leq T_{0}$. In fact, suppose that (3.44) is true for some $j \geq 0$. Firstly, the Sobolev inequality shows

$$
\sup _{0 \leq t \leq T_{0}, x \in \mathbb{R}^{2}}\left|c^{j}(t, x)\right| \leq C M .
$$

Then, the zero-order energy estimate on $c^{j+1}$ gives

$$
\begin{gathered}
\frac{1}{2} \frac{d}{d t}\left\|c^{j+1}\right\|^{2}+\mu\left\|\nabla c^{j+1}\right\|^{2} \leq \int_{\mathbb{R}^{2}}\left|u c^{j} \cdot \nabla c^{j+1}\right| d x+\int_{\mathbb{R}^{2}}\left|k\left(c^{j}\right) \widetilde{n} c^{j+1}\right| d x \\
\leq \frac{\mu}{2}\left\|\nabla c^{j+1}\right\|^{2}+C\|u\|_{L^{\infty}}^{2}\left\|c^{j}\right\|^{2}+\left\|c^{j+1}\right\|^{2}+C\|\widetilde{n}\|^{2},
\end{gathered}
$$


which from the Gronwall inequality leads to

$$
\sup _{0 \leq t \leq T_{0}}\left\|c^{j+1}(t)\right\|^{2} \leq e^{2 T_{0}}\left(\left\|c_{0}\right\|^{2}+C M\|u\|_{L^{\infty}\left(0, T ; H^{2}\right)}^{2} T_{0}+C\|\widetilde{n}\|_{L^{2}\left(0, T_{0} ; L^{2}\right)}^{2}\right)
$$

and further

$$
\int_{0}^{T_{0}}\left\|\nabla c^{j+1}(s)\right\|^{2} d s \leq\left(1+2 e^{2 T_{0}}\right)\left(\left\|c_{0}\right\|^{2}+C M\|u\|_{L^{\infty}\left(0, T ; H^{2}\right)}^{2} T_{0}+C\|\widetilde{n}\|_{L^{2}\left(0, T_{0} ; L^{2}\right)}^{2}\right) .
$$

For the first-order energy estimate, it is straightforward to get

$$
\left\|\nabla c^{j+1}(t)\right\|^{2}+\lambda \int_{0}^{t}\left\|\nabla^{2} c^{j+1}(s)\right\|^{2} d s \leq\left\|\nabla c_{0}\right\|^{2}+C M\|u\|_{L^{\infty}\left(0, T ; H^{2}\right)}^{2} T_{0}+C\|\widetilde{n}\|_{L^{2}\left(0, T_{0} ; L^{2}\right)}^{2}
$$

for any $0 \leq t \leq T_{0}$. The second-order energy estimate gives

$$
\frac{1}{2} \frac{d}{d t}\left\|\nabla^{2} c^{j+1}\right\|^{2}+\lambda\left\|\nabla^{3} c^{j+1}\right\|^{2} \leq C\left\|\nabla\left(u \cdot \nabla c^{j}\right)\right\|^{2}+C\left\|\nabla\left(k\left(c^{j}\right) \widetilde{n}\right)\right\|^{2},
$$

where it further holds that

$$
\begin{aligned}
\left\|\nabla\left(u \cdot \nabla c^{j}\right)\right\|^{2} & \leq C\left\|\nabla u \nabla c^{j}\right\|^{2}+C\left\|u \cdot \nabla^{2} c^{j}\right\|^{2} \leq C\|\nabla u\|^{2}\left\|\nabla c^{j}\right\|_{L^{\infty}}^{2}+C\|u\|_{L^{\infty}}^{2}\left\|\nabla^{2} c^{j}\right\|^{2} \\
& \leq C_{\kappa}\|\nabla u\|^{4}\left\|\nabla c^{j}\right\|^{2}+\kappa\left\|\nabla^{3} c^{j}\right\|^{2}+C\|u\|_{H^{2}}^{2}\left\|\nabla^{2} c^{j}\right\|^{2}
\end{aligned}
$$

for some small constant $\kappa>0$ to be chosen later, and

$$
\left\|\nabla\left(k\left(c^{j}\right) \widetilde{n}\right)\right\|^{2} \leq C\|\nabla \widetilde{n}\|^{2}+C\left\|\nabla c^{j}\right\|_{L^{4}}^{2}\|\widetilde{n}\|_{L^{4}}^{2} \leq C\|\nabla \widetilde{n}\|^{2}+C\left\|\nabla c^{j}\right\|_{H^{1}}^{2}\|\widetilde{n}\|_{H^{1}}^{2} .
$$

Then, it follows that

$$
\begin{aligned}
\left\|\nabla^{2} c^{j+1}(t)\right\|^{2}+\lambda \int_{0}^{t}\left\|\nabla^{3} c^{j+1}\right\|^{2} d s \leq & \left\|\nabla^{2} c_{0}\right\|^{2}+C_{\kappa} M\left(1+\|u\|_{L^{\infty}\left(0, T_{0} ; H^{2}\right)}^{4}\right) T_{0} \\
& +C(1+M)\|\widetilde{n}\|_{L^{2}\left(0, T_{0} ; H^{1}\right)}^{2}+C \kappa M .
\end{aligned}
$$

for any $0 \leq t \leq T_{0}$. Collecting all the above estimates, using $\widetilde{n} \in Y(0, T)$ and the estimate (3.41), and then choosing properly small constants $\lambda>0, \kappa>0, T_{0}>0$ and large constant $M>0,(3.44)$ is true for $j+1$ and hence it holds for all $j \geq 0$ by induction. Now, one can extract a convergent subsequence of $\left(c^{j}\right)_{j \geq 0}$ so that its limit function $c$ is indeed a solution to the Cauchy problem. The uniqueness also holds from the Gronwall inequality. Iterating the above process, we prove the existence and uniqueness of solutions over the whole time interval $[0, T]$ if additionally $c_{0} \in H^{2}\left(\mathbb{R}^{2}\right)$.

The rest is to derive uniform a priori estimates on $c$ if $c_{0} \in H^{1}\left(\mathbb{R}^{2}\right) \cap L^{\infty}\left(\mathbb{R}^{2}\right)$. Similarly as before, if $\left\|c_{0}\right\|_{L^{\infty}}$ is small, then from (3.43),

$$
\frac{1}{2} \frac{d}{d t}\|c\|_{H^{1}}^{2}+\lambda\|\nabla c\|_{H^{1}}^{2} \leq C\left\|c_{0}\right\|_{L^{\infty}}\left(\|\nabla \widetilde{n}\|^{2}+\|\nabla u\|^{2}\right)
$$

which gives

$$
\|c(t)\|_{H^{1}}^{2}+\lambda \int_{0}^{t}\|\nabla c\|_{H^{1}}^{2} d s \leq C\left\|c_{0}\right\|_{L^{\infty}} \int_{0}^{T}\left(\|\nabla \widetilde{n}\|^{2}+\|\nabla u\|^{2}\right) d s
$$

for all $0 \leq t \leq T$. If $\left\|c_{0}\right\|_{L^{4}}$ is small, then from (3.43), we use the following estimate

$$
\frac{1}{2} \frac{d}{d t}\|c\|_{H^{1}}^{2}+\lambda\|\nabla c\|_{H^{1}}^{2} \leq C\left\|c_{0}\right\|_{L^{\infty}}\|\nabla u\|^{2}+C\left\|c_{0}\right\|_{L^{4}}^{2}\|\nabla \widetilde{n}\|^{2}
$$

which yields

$$
\|c(t)\|_{H^{1}}^{2}+\lambda \int_{0}^{t}\|\nabla c\|_{H^{1}}^{2} d s \leq C\left\|c_{0}\right\|_{L^{\infty}} \int_{0}^{T}\|\nabla u\|^{2} d s+\left\|c_{0}\right\|_{L^{4}}^{2} \int_{0}^{T}\|\nabla \widetilde{n}\|^{2} d s
$$


for all $0 \leq t \leq T$. Notice that one has

$$
c \in C\left([0, T] ; H^{1}\right) \cap L^{2}\left(0, T ; H^{2}\right) \cap L^{\infty}\left((0, T) \times \mathbb{R}^{2}\right) .
$$

(iii) Solve $n=: \mathcal{T}_{3}[\widetilde{n}]$ to be the solution to the Cauchy problem

$$
\left\{\begin{array}{l}
\partial_{t} n+u \cdot \nabla n=\delta \Delta n-\nabla \cdot(\chi(c) n \nabla c), \\
\left.n\right|_{t=0}=n_{0}
\end{array}\right.
$$

where $u=\mathcal{T}_{1}[\widetilde{n}]$ and $c=\mathcal{T}_{2}[\widetilde{n}]$ are given in (i) and (ii), respectively. In fact, under the conditions (3.42) and (3.47) for $u$ and $c$, the existence and uniqueness for the linear second-order parabolic equation (3.48) follows from the similar proof as in (ii), and thus their proof is omitted for simplicity. Here, let us give some uniform estimate of $n$ for compactness argument later. Firstly, from the Maximum principle and $n_{0} \geq 0$, one has $n(t, x) \geq 0$ for any $t \geq 0$ and $x \in \mathbb{R}^{2}$. Moreover, it holds that

$$
\begin{aligned}
\frac{1}{2} \frac{d}{d t}\|n\|^{2}+\delta\|\nabla n\|^{2} & =\int_{\mathbb{R}^{2}} n \chi(c) \nabla c \cdot \nabla n d x \leq C\|n\|_{L^{p}}\|\nabla c\|_{L^{\frac{2 p}{p-2}}}\|\nabla n\| \\
& \leq C\|n\|^{\frac{2}{p}}\|\nabla c\|_{L^{\frac{2 p}{p-2}}}\|\nabla n\|^{\frac{2 p-2}{p}} \\
& \leq \frac{\delta}{2}\|\nabla n\|^{2}+C_{p}\|\nabla c\|_{L^{\frac{2 p}{p-2}}}^{p}\|n\|^{2} \\
& \leq \frac{\delta}{2}\|\nabla n\|^{2}+C_{p}\|\nabla c\|^{p-2}\left\|\nabla^{2} c\right\|^{2}\|n\|^{2}
\end{aligned}
$$

for $2<p<\infty$, which from the Gronwall inequality and (3.47) implies

$$
n \in C\left([0, T] ; L^{2}\right) \cap L^{2}\left(0, T ; H^{1}\right)
$$

and

$$
\begin{gathered}
\|n(t)\|^{2}+\lambda \int_{0}^{t}\|\nabla n\|^{2} d s \leq\left\|n_{0}\right\|^{2} \\
+C_{p}\left\|n_{0}\right\|^{2} e^{C_{p} \sup _{0 \leq s \leq T}\|\nabla c(s)\|^{p-2} \int_{0}^{T}\left\|\nabla^{2} c\right\|^{2} d s} \sup _{0 \leq s \leq T}\|\nabla c(s)\|^{p-2} \int_{0}^{T}\left\|\nabla^{2} c\right\|^{2} d s .
\end{gathered}
$$

The moment estimate $|x|^{2} n \in L^{\infty}\left(0, T ; L^{1}\left(\mathbb{R}^{2}\right)\right)$ follows from

$$
\begin{aligned}
\frac{d}{d t} \int_{\mathbb{R}^{2}}|x|^{2} n(t, x) d x & =4 \delta \int_{\mathbb{R}^{2}} n d x+2 \int_{\mathbb{R}^{2}} n u \cdot x d x-2 \int_{\mathbb{R}^{2}} \chi(c) n \nabla c \cdot x d x \\
& \leq 4 \delta\left\|n_{0}\right\|_{L^{1}}+\left(\|u \sqrt{n}\|^{2}+\|\chi(c) \nabla c \sqrt{n}\|^{2}\right) \int_{\mathbb{R}^{2}}|x|^{2} n(t, x) d x,
\end{aligned}
$$

and

$$
\|u \sqrt{n}\|^{2} \leq\|u\|_{L^{4}}^{2}\|\sqrt{n}\|_{L^{4}}^{2} \leq C\|u\|_{H^{1}}^{2}\|n\|, \quad\|\chi(c) \nabla c \sqrt{n}\|^{2} \leq C\|\nabla c\|_{H^{1}}^{2}\|n\|
$$

together with the Gronwall inequality and (3.42), (3.47) and (3.49). Finally, by dual argument, it is also straightforward to verify $\partial_{t} n \in L^{2}\left(0, T ; V^{\prime}\right)$.

Now, we are in a position to complete the proof of Proposition 3.1. Define $\mathcal{T}=\mathcal{T}_{3}$ by $n=\mathcal{T}_{3}[\widetilde{n}]$. From (i)(ii)(iii) above,

$$
\mathcal{T}: Y(0, T) \subset L^{2}(0, T ; H) \rightarrow Y(0, T) \subset L^{2}(0, T ; H)
$$

is indeed well-defined and further $\mathcal{T}$ is continuous over $Y(0, T)$. Recall a compactness argument by the following 
Lemma 3.3 (Aubin-Lions lemma). Let $T>0$ and $1<p<\infty$, and let $H$ be a Banach space and $V \subseteq H$ be a subspace compactly imbedded in $H$. If the sequence $\left(f_{j}\right)_{j \geq 1}$ is bounded in $L^{p}(0, T ; V)$ and $\left(\partial_{t} f_{j}\right)_{j \geq 1}$ is bounded in $L^{p}\left(0, T ; V^{\prime}\right)$ uniformly in $j \geq 1$, then $\left(f_{j}\right)_{j \geq 1}$ is relatively compact in $L^{p}(0, T ; H)$.

In our case, $p=2$ and the functional spaces $H, V, V^{\prime}$ defined at the beginning satisfy the assumption of Lemma 3.3. Therefore, from the above lemma, one can apply Schauder fixed point theorem to a ball in $Y(0, T)$, and hence the Cauchy problem admits one solution in $Y(0, T)$. Notice that the ball can be mapped into itself by $\mathcal{T}$ as long as the radius of the ball is sufficiently large and the smallness of either $M_{\phi},\left\|c_{0}\right\|_{L^{4}}$ or $\left\|c_{0}\right\|_{L^{\infty}}$ is assumed. In fact, for the bound of $n$ in $L^{2}\left(0, T ; H^{1}\left(\mathbb{R}^{2}\right)\right)$, this follows from estimates (3.41), (3.45) or (3.46), and (3.50). For the bound of $|x|^{2} n$ in $L^{\infty}\left(0, T ; L^{1}\left(\mathbb{R}^{2}\right)\right)$, it follows further from (3.51) and (3.52) and Gronwall inequality. It similarly holds for the bound of $\partial_{t} n$ in $L^{2}\left(0, T ; V^{\prime}\right)$. The rest conclusions in Proposition 3.1 directly follow from (i)(ii)(iii). This completes the proof of Proposition 3.1.

Step 2 A priori estimates on the regularized solutions uniformly in the regularization parameter. Under assumptions $(\mathbf{A})(\mathbf{B 1})(\mathbf{B} 2)(\mathbf{B 3})$ or $(\mathbf{A})(\mathbf{C 1})(\mathbf{C 2})(\mathbf{C} 3)$, one can apply Lemma 3.1 and Lemma 3.2 respectively to the regularized solution $\left(n^{\epsilon}, c^{\epsilon}, u^{\epsilon}\right)$ with initial data satisfying (3.34), (3.35), (3.36) (3.37) and (3.38) so that (3.3) (3.4), (3.5) and (3.6) hold for $\left(n^{\epsilon}, c^{\epsilon}, u^{\epsilon}\right)$ :

- $n^{\epsilon}(t, x) \geq 0, c^{\epsilon}(t, x) \geq 0, t \geq 0, x \in \mathbb{R}^{2}$, and for any $T>0$,

$$
\left\{\begin{array}{l}
n^{\epsilon}\left(1+|x|+\left|\ln n^{\epsilon}\right|\right) \in L^{\infty}\left(0, T ; L^{1}\left(\mathbb{R}^{2}\right)\right), \nabla \sqrt{n^{\epsilon}} \in L^{2}\left(0, T ; L^{2}\left(\mathbb{R}^{2}\right)\right), \\
c^{\epsilon} \in L^{\infty}\left(0, T ; L^{1}\left(\mathbb{R}^{2}\right) \cap L^{\infty}\left(\mathbb{R}^{2}\right) \cap H^{1}\left(\mathbb{R}^{2}\right)\right), \nabla c^{\epsilon} \in L^{2}\left(0, T ; L^{2}\left(\mathbb{R}^{2}\right)\right), \\
\sqrt{n^{\epsilon}}\left|\nabla c^{\epsilon}\right| \in L^{2}\left(0, T ; L^{2}\left(\mathbb{R}^{2}\right)\right), \\
u^{\epsilon} \in L^{\infty}\left(0, T ; L^{2}\left(\mathbb{R}^{2}\right)\right), \nabla u^{\epsilon} \in L^{2}\left(0, T ; L^{2}\left(\mathbb{R}^{2}\right)\right),
\end{array}\right.
$$

where all bounds in the corresponding spaces are independent of $\epsilon$. Furthermore, in the case of weak force, by Lemma 3.1 and (3.27),

$$
\nabla c^{\epsilon} \in L^{2}\left(0, T ; L^{4}\left(\mathbb{R}^{2}\right)\right),
$$

while in the case of small $c_{0}$, from Lemma 3.2 ,

$$
\nabla c^{\epsilon} \in L^{2}\left(0, T ; H^{1}\left(\mathbb{R}^{2}\right)\right) .
$$

- $\forall \varphi \in C_{0}^{\infty}\left([0, \infty) \times \mathbb{R}^{2}\right)$,

$$
\begin{gathered}
\int_{0}^{\infty} \int_{\mathbb{R}^{2}} n^{\epsilon}\left(\partial_{t} \varphi+\delta \Delta \varphi\right) d x d t+\int_{0}^{\infty} \int_{\mathbb{R}^{2}} n^{\epsilon} u^{\epsilon} \cdot \nabla \varphi d x d t \\
+\int_{0}^{\infty} \int_{\mathbb{R}^{2}} \chi\left(c^{\epsilon}\right) n^{\epsilon} \nabla c^{\epsilon} \cdot \nabla \varphi d x d t+\int_{\mathbb{R}^{2}} n_{0}^{\epsilon}(x) \varphi(0, x) d x=0, \\
\int_{0}^{\infty} \int_{\mathbb{R}^{2}} c^{\epsilon}\left(\partial_{t} \varphi+\mu \Delta \varphi\right) d x d t+\int_{0}^{\infty} \int_{\mathbb{R}^{2}} c^{\epsilon} u^{\epsilon} \cdot \nabla \varphi d x d t \\
-\int_{0}^{\infty} \int_{\mathbb{R}^{2}} k\left(c^{\epsilon}\right) n^{\epsilon} \varphi d x d t+\int_{\mathbb{R}^{2}} c_{0}^{\epsilon}(x) \varphi(0, x) d x=0,
\end{gathered}
$$

and $\forall \widetilde{\varphi} \in C_{0}^{\infty}\left([0, \infty) \times \mathbb{R}^{2}\right)$ with $\nabla \cdot \widetilde{\varphi}=0$,

$$
\int_{0}^{\infty} \int_{\mathbb{R}^{2}} u^{\epsilon} \cdot\left(\partial_{t} \widetilde{\varphi}+\nu \Delta \widetilde{\varphi}\right) d x d t-\int_{0}^{\infty} \int_{\mathbb{R}^{2}} n^{\epsilon} \nabla \phi \cdot \widetilde{\varphi} d x d t+\int_{\mathbb{R}^{2}} u_{0}^{\epsilon}(x) \cdot \widetilde{\varphi}(0, x) d x=0 .
$$

Step 3 Passing to the limit. Let $\epsilon=\epsilon_{j}$ with $\epsilon_{j} \searrow 0$ as $j \rightarrow \infty$. From Step 2, one has that up to a subsequence, $n^{\epsilon}, c^{\epsilon}, u^{\epsilon}$, respectively, converge to some functions $n, c, u$ in distributions. In what 
follows, let us verify that $(n, c, u)$ is indeed a weak solution to the Cauchy problem in the sense of Definition 3.1.

Firstly, those a priori bounds independent of $\epsilon$ in (3.53) yield the weak convergence:

$$
\begin{gathered}
n^{\epsilon} \rightarrow n \text { in w- } L^{p}\left(0, T ; L^{1}\left(\mathbb{R}^{2}\right)\right), \\
c^{\epsilon} \rightarrow c \text { in } \mathrm{w}-L^{p}\left((0, T) \times \mathbb{R}^{2}\right) \cap L^{p}\left(0, T ; H^{1}\left(\mathbb{R}^{2}\right)\right), \\
u^{\epsilon} \rightarrow u \text { in w- } L^{p}\left(0, T ; L^{2}\left(\mathbb{R}^{2}\right)\right),
\end{gathered}
$$

for any $1 \leq p<\infty$. Since $\left(c^{\epsilon}\right)_{\epsilon>0}$ is uniformly bounded in $H^{1}\left(\mathbb{R}^{2}\right)$ for a.e. $t \in[0, T]$, then up to a subsequence, $\left(c^{\epsilon}\right)_{\epsilon>0}$ strongly converges to $c$ in $L^{2}\left(\mathbb{R}_{\text {loc }}^{2}\right)$ over a rational dense subset of $[0, T]$. Since $\left\|c^{\epsilon}\right\|$ is continuous in $t$, then by re-defining $c,\left(c^{\epsilon}\right)_{\epsilon>0}$ strongly converges to $c$ in $L^{2}\left(\mathbb{R}_{\text {loc }}^{2}\right)$ over $[0, T]$. Since $\left(c^{\epsilon}\right)_{\epsilon>0}$ is also uniformly bounded in $L^{p}\left(\mathbb{R}^{2}\right)$ for $1 \leq p \leq \infty$, then by Hölder inequality, $\left(c^{\epsilon}\right)_{\epsilon>0}$ strongly converges to $c$ in $L^{p}\left(\mathbb{R}_{\text {loc }}^{2}\right)$ and hence in $L^{p}\left((0, T) \times \mathbb{R}_{\text {loc }}^{2}\right)$ for $1 \leq p<\infty$. For the convergence of $\left(n^{\epsilon}\right)_{\epsilon>0}$, since $\left(\sqrt{n^{\epsilon}}\right)_{\epsilon>0}$ is uniformly bounded in $H^{1}\left(\mathbb{R}^{2}\right)$ for a.e. $t \in[0, T]$, then similarly before, up to a subsequence, $\left(\sqrt{n^{\epsilon}}\right)_{\epsilon>0}$ strongly converges to $\sqrt{\bar{n}}$ in $L^{2}\left(\mathbb{R}_{\text {loc }}^{2}\right)$ over $[0, T]$. Since

$$
\begin{aligned}
\int_{\Omega_{c}}\left|n^{\epsilon}-\bar{n}\right| d x & =\int_{\Omega_{c}}\left|\left(\sqrt{n^{\epsilon}}\right)^{2}-(\sqrt{\bar{n}})^{2}\right| d x \\
& =\int_{\Omega_{c}}\left|\sqrt{n^{\epsilon}}+\sqrt{\bar{n}}\right| \cdot\left|\sqrt{n^{\epsilon}}-\sqrt{\bar{n}}\right| d x \\
& \leq C\left(\left\|n^{\epsilon}\right\|_{L^{1}}+\|\bar{n}\|_{L^{1}}\right)\left\|\sqrt{n^{\epsilon}}-\sqrt{\bar{n}}\right\|_{L^{2}\left(\Omega_{c}\right)}
\end{aligned}
$$

for any compact subset $\Omega_{c}$ of $\mathbb{R}^{2},\left(n^{\epsilon}\right)_{\epsilon>0}$ strongly converges to $\bar{n}=n$ in $L^{1}\left((0, T) \times \mathbb{R}_{\text {loc }}^{2}\right)$. Notice that from Sobolev inequality,

$$
\int_{\mathbb{R}^{2}}\left|n^{\epsilon}\right|^{p} d x=\left\|\sqrt{n^{\epsilon}}\right\|_{L^{2 p}}^{2 p} \leq C(p)\left\|\sqrt{n^{\epsilon}}\right\|^{2}\left\|\nabla \sqrt{n^{\epsilon}}\right\|^{2(p-1)} \leq C\left(p,\left\|n_{0}\right\|_{L^{1}}\right)\left\|\nabla \sqrt{n^{\epsilon}}\right\|^{2(p-1)},
$$

for $1 \leq p<\infty$, which implies that $\left(n^{\epsilon}\right)_{\epsilon>0}$ is uniformly bounded in $L^{\frac{p}{p-1}}\left(0, T ; L^{p}\left(\mathbb{R}^{2}\right)\right)$ for $1 \leq p<\infty$. Further using the uniform boundedness of $\left(n^{\epsilon}\right)_{\epsilon>0}$ in $L^{\infty}\left(0, T ; L^{1}\left(\mathbb{R}^{2}\right)\right)$, it follows that $\left(n^{\epsilon}\right)_{\epsilon>0}$ is actually uniformly bounded in $L^{p}\left((0, T) \times \mathbb{R}^{2}\right)$ for $1 \leq p \leq 2$ and thus $\left(n^{\epsilon}\right)_{\epsilon>0}$ strongly converges to $n$ in $L^{p}\left((0, T) \times \mathbb{R}_{\text {loc }}^{2}\right)$ for $1 \leq p \leq 2$. Similarly, it follows that $\left(u^{\epsilon}\right)_{\epsilon>0}$ strongly converges to $u$ in $L^{2}\left((0, T) \times \mathbb{R}_{\text {loc }}^{2}\right)$. These are enough to pass to the limit for all nonlinear terms in (3.54), (3.55) and (3.56). Finally, due to the weak lower semi-continuity of norms and the convexity of functionals $n \mapsto \int_{\mathbb{R}^{2}} n \ln n d x$ and $n \mapsto \int_{\mathbb{R}^{2}}|\nabla \sqrt{n}|^{2} d x$, one can obtain all bounds for the limit functions $n, c, u$ in the corresponding functional spaces given in (3.53). Therefore, $(n, c, u)$ satisfies all conditions in Definition 3.1. This completes the proof of Theorem 3.1.

\section{Acknowledgement}

This research is supported by Award No. KUK-I1-007-43, made by King Abdullah University of Science and Technology (KAUST). R.-J. Duan would like to thank RICAM for its support during the postdoctoral studies of the year 2008-09. A. Lorz would like to acknowledge support by KAUST. P. Markowich acknowledges support from his Royal Society Wolfson Research Merit Award. The authors would like to thank the anonymous referees for their valuable comments to improve the current results so much.

\section{References}

[1] Blanchet, A., Carrillo, J. A., Masmoudi, N. (2008). Infinite time aggregation for the critical Patlak-Keller-Segel model in $\mathbb{R}^{2}$. Comm. Pure Appl. Math. 61(10):1449-1481. 
Chemotaxis-Fluid Equations

[2] Blanchet, A., Dolbeault, J., Perthame, B. (2006). Two-dimensional Keller-Segel model: Optimal critical mass and qualitative properties of the solutions. Electronic Journal of Differential Equations 44:1-32.

[3] Boudin, L., Desvillettes, L., Grandmont, C., Moussa, A. Global existence of solutions for the coupled Vlasov and Navier-Stokes equations, to appear in Differential and Integal Equations.

[4] Caflisch, R., Papanicolaou, G.C. (1983). Dynamic theory of suspensions with Brownian effects. SIAM J. Appl. Math. 43(4):885-906.

[5] Calvez, V., Corrias, L. (2008). The Parabolic-parabolic Keller-Segel Model in $\mathbb{R}^{2}$. Comm. Math. Sci. 6:417- 447.

[6] Carrillo, J. A., Goudon, T. (2006). Stability and asymptotic analysis of a fluid-particle interaction model. Communications in Partial Differential Equations 31:1349-1379.

[7] Chalub, F., Dolak-Struss, Y., Markowich, P., Oelz, D., Schmeiser, C., Soreff, A. (2006). Model hierarchies for cell aggregation by chemotaxis. Math. Models Methods Appl. Sci. 16(7):1173-1197.

[8] Chalub, F., Markowich, P., Perthame, B., Schmeiser, C. (2004). Kinetic models for chemotaxis and their drift-diffusion limits. Monatsh. Math. 142(1-2):123-141.

[9] Corrias, L., Perthame, B., Zaag, H. (2003). A chemotaxis model motivated by angiogenesis. C. R. Math. Acad. Sci. Paris 336(2):141-146.

[10] Corrias, L., Perthame, B., Zaag, H. (2004). Global solutions of some chemotaxis and angiogenesis systems in high space dimensions. Milan J. Math. 72:1-28.

[11] Duan, R.-J., Ukai, S., Yang, T., Zhao, H.-J. (2008). Optimal decay estimates on the linearized Boltzmann equation with time dependent force and their applications. Comm. Math. Phys. 277:189-236.

[12] Fontelos, M. A., Friedman, A., Hu, B. (2002). Mathematical analysis of a model for the initiation of angiogenesis. SIAM J. Math. Anal. 33:1330-1355.

[13] Goudon, T. (2005). Hydrodynamic limit for the Vlasov-Poisson-Fokker-Planck system: analysis of the two-dimensional case. Math. Mod. Meth. Appl. Sci. 15:737-752.

[14] Goudon, T., He, L., Moussa, A., Zhang P. The Navier-Stokes-Vlasov-Fokker-Planck system near equilibrium, preprint.

[15] Guarguaglini, F. R., Natalini, R. (2003). Global existence of solutions to a nonlinear model of sulphation phenomena in calcium carbonate stones. To appear.

[16] Hamdache, K. (1998). Global existence and large time behaviour of solutions for the VlasovStokes equations. Japan J. Indust. Appl. Math. 15(1):51-74.

[17] Hillesdon, A. J., Pedley, T. J., Kessler, J. O. (1995). The development of concentration gradients in a suspension of chemotactic bacteria. Bull. Math. Bio.57:299-344.

[18] Horstmann, D. (2003). From 1970 until present: the Keller-Segel model in chemotaxis and its consequences. I. Jahresber. Deutsch. Math.-Verein. 105(3):103-165.

[19] Horstmann, D. (2004). From 1970 until present: the Keller-Segel model in chemotaxis and its consequences. II. Jahresber. Deutsch. Math.-Verein. 106(2):51-69.

[20] Kawashima, S., Nishibata, S., Nishikawa, M. (2004). $L^{p}$ energy method for multi-dimensional viscous conservation laws and application to the stability of planar waves. J. Hyperbolic Differ. Equ. 1:581-603.

[21] Keller, E. F., Segel, L. A. (1970). Initiation of slime mold aggregation viewed as an instability. J. Theor. Biol. 26:399-415. 
[22] Ladyzhenskaya, O. A. (1969). The Mathematical Theory of Viscous Incompressible Flow. Second English edition, revised and enlarged. New York-London-Paris: Science Publishers.

[23] Lions, P.-L. (1996). Mathematical Topics in Fluid Mechanics. Vol. 1. Incompressible models. Oxford Lecture Series in Mathematics and its Applications, 3. Oxford Science Publications. New York: Oxford University Press.

[24] Markowich, P. (1984). A Singular Perturbation Analysis of the Fundamental Semiconductordevice equations. SIAM J. App. Math. 44:896-928.

[25] Mellet, A., Vasseur, A. (2009). Asymptotic analysis for a Vlasov-Fokker-Planck/Compressible Navier-Stokes system of equations. to appear in Comm. Math. Phys..

[26] Mellet, A., Vasseur, A. (2007). Global weak solutions for a Vlasov-Fokker-Planck/Navier-Stokes system of equations, Math. Models Methods Appl. Sci. 17:1039-1063.

[27] Metcalfe, A. M., Pedley, T. J. (1998). Bacterial bioconvection: weakly nonlinear theory for pattern selection, J. Fluid Mech., 370: 249-270.

[28] Pedley, T. J., Kessler, J. O. (1992). Hydrodynamic phenomena in suspensions of swimming microorganisms, Annu. Rev. Fluid Mech. 24: 313-358.

[29] Rascle, M. (1980). On a system of non-linear strongly coupled partial differential equations arising in biology. Lectures Notes in Math. 846 Everitt and Sleeman eds., New York: Springer-Verlag.

[30] Tuval, I., Cisneros, L., Dombrowski, C., Wolgemuth, C. W., Kessler, J.O., Goldstein, R.E. (2005). Bacterial swimming and oxygen transport near contact lines. Proceedings of the National Academy of Sciences 102(7):2277-2282.

[31] Tupchiev, V. A., Fomina, N. A. (2004). On the well-posedness and stabilization of solutions of boundary value problems of chemotaxis. (Russian) Zh. Vychisl. Mat. Mat. Fiz. 44(5):917-943; translation in Comput. Math. Math. Phys. 44(5):868-893. 